\title{
Cambio climático y mercados de carbono: repercusiones para los países en desarrollo
}

\author{
Carlos Ludeña, Carlos de Miguel y Andrés Schuschny
}

RESUMEN

Si bien el Protocolo de Kyoto proporcionó un marco para reducir las emisiones de gases de efecto invernadero de las naciones industrializadas, las actuales negociaciones sobre cambio climático anticipan futuros compromisos de los principales emisores de carbono entre los países en desarrollo. Se utiliza aquí una versión actualizada del modelo de equilibrio general del Proyecto de Análisis del Comercio Global (GTAP-E) para analizar los efectos económicos de la reducción de las emisiones de carbono bajo diferentes hipótesis de comercio de derechos de emisión. La participación de países en desarrollo como China y la India reduciría los costos de ese comercio. Las repercusiones en América Latina varían si se trata de un país exportador o importador de energía y si se reducen o no las emisiones estadounidenses. Los efectos en el bienestar podrían ser negativos según el sistema de comercio de derechos adoptado y los socios comerciales.

PALABRAS CLAVE

CLASIFICACIÓN JEL

AUTORES
Cambio climático, acuerdos sobre el medioambiente, anhídrido carbónico, mercados, derechos negociables de emisión, aspectos económicos, estadísticas ambientales, países en desarrollo, América Latina

C68, D58, H23, Q52, Q54, Q56

Carlos Ludeña es economista del cambio climático de la División de Cambio Climático y Sostenibilidad del Banco Interamericano de Desarrollo (BID). carlosl@iadb.org

Carlos de Miguel es jefe de la Unidad de Políticas para el Desarrollo Sostenible de la División de Desarrollo Sostenible y Asentamientos Humanos de la Comisión Económica para América Latina y el Caribe (CEPAL). carlos.demiguel@cepal.org

Andrés Schuschny es funcionario de la Unidad de Recursos Naturales y Energía de la División de Recursos Naturales e Infraestructura de la Comisión Económica para América Latina y el Caribe (CEPAL). andres. schuschny@cepal.org 


\section{I}

\section{Introducción}

El cambio climático es uno de los mayores retos que enfrenta la humanidad en el siglo XXI. La comunidad científica está de acuerdo en que el planeta se está calentando con la mayor rapidez establecida en 10.000 años, y que este cambio en la temperatura ha sido causado por el aumento del dióxido de carbono $\left(\mathrm{CO}_{2}\right)$ y otros gases de efecto invernadero en la atmósfera del planeta, sobre todo en los últimos 100 años. Este incremento se debe especialmente a las actividades antropogénicas. El nivel de gases de efecto invernadero en la atmósfera equivale en la actualidad a casi 400 partes por millón (ppm) de $\mathrm{CO}_{2}$, en comparación con solo 280 ppm antes de la Revolución Industrial. De mantenerse la tendencia actual, se prevé que esa cifra crecerá más de $2 \mathrm{ppm}$ por año (Stern, 2007). Sobre la base de la duplicación de los niveles preindustriales de gases de efecto invernadero, en la mayoría de los modelos climáticos se proyecta una elevación de las temperaturas medias globales de alrededor de $2{ }^{\circ} \mathrm{C}$ a $5{ }^{\circ} \mathrm{C}$ en las próximas décadas. Por ejemplo, un nivel de estabilización de 450 ppm de $\mathrm{CO}_{2}$ equivalente supondría una probabilidad del $78 \%$ de un incremento de la temperatura superior a $2{ }^{\circ} \mathrm{C}$ y una probabilidad del $18 \%$ de un aumento de $3{ }^{\circ} \mathrm{C}$ o superior (Stern, 2007). Entre otras consecuencias, se prevén cambios en los patrones de precipitación, la reducción de las masas de hielo y los depósitos de nieve mundiales, el elevamiento del nivel del mar y modificaciones en la intensidad y frecuencia de los fenómenos climáticos extremos (IPCC, 2007). El cambio climático afectará en forma considerable a la actividad económica, la población y los ecosistemas, y será un factor que tendrá un papel fundamental en la determinación de las características del desarrollo económico en este siglo.

Para limitar el probable ascenso de las temperaturas se deben estabilizar y reducir los niveles de $\mathrm{CO}_{2}$ y otros gases de efecto invernadero. Esa reducción no puede lograrse con los esfuerzos de una sola nación o gobierno, sino que requiere el compromiso de todos los gobiernos del mundo.

La Convención Marco de las Naciones Unidas sobre el Cambio Climático (CMNUCC), el Protocolo de Kyoto y otros tratados constituyen un marco para fomentar la cooperación internacional con respecto a este tema. En el Protocolo de Kyoto (CMNUCC, 1997) se estableció la obligación legal para algunos países industrializados (denominados países del Anexo I) de mitigar sus emisiones de gases de efecto invernadero y se delinearon mecanismos como el comercio de derechos de emisión, u otros con miras a un desarrollo limpio y la aplicación conjunta a objeto de ayudar a aquellos países en ese cometido. En la actualidad, el Protocolo de Kyoto de la CMNUCC comprende 193 partes (192 Estados y una organización de integración económica regional). La participación en las emisiones de gases de efecto invernadero de las partes incluidas en el Anexo I varía entre el $35 \%$ y el $40 \%$ del total, dependiendo de si se incluyen las provenientes del cambio de uso del suelo y del sector forestal.

Los países que no figuran en el Anexo I, incluidos los de América Latina y el Caribe, no tienen restricciones o compromisos relativos a las emisiones de gases de efecto invernadero además de lo establecido en acuerdos voluntarios. Sin embargo, cuentan con incentivos financieros para implementar proyectos de reducción de dichas emisiones y recibir créditos de carbono, que a su vez pueden vender a los países del Anexo I a fin de ayudarlos a alcanzar sus metas. Por otra parte, debido a la escala de la reducción de las emisiones requerida, es probable que los acuerdos multilaterales para ser realmente eficaces deban involucrar tanto a países desarrollados como en desarrollo. En consecuencia, se espera que las recientes y futuras Conferencias de las Naciones Unidas sobre el Cambio Climático ofrezcan una respuesta internacional eficaz al cambio climático mediante nuevos compromisos para los países del Anexo I del Protocolo de Kyoto y los países de la CMNUCC en general.

De esta manera, en las negociaciones relativas al segundo período de compromiso (posterior a 2012), en el marco del Protocolo, se han introducido variantes en el régimen mundial, que no solo profundizan las obligaciones de los países desarrollados, sino que podrían dar lugar a compromisos para diferentes sectores o actividades en todo el mundo y respecto de los países en desarrollo, basados en criterios de responsabilidad y capacidad (Samaniego, 2009). Stern (2008) estima que el objetivo de reducir las emisiones un $100 \%$ antes de 2050 solo se alcanzará si los países en desarrollo logran un recorte del $28 \%$ en 
sus emisiones per cápita en ese plazo. La participación de dichos países también disminuirá el costo de recortar las emisiones. De la Torre, Fajnzylber y Nash (2009) afirman que una solución eficaz a nivel mundial solo es posible si la disminución de las emisiones de gases de efecto invernadero se logra en los países donde el costo de su reducción es bajo, y no necesariamente en aquellos cuyo nivel de emisiones es más alto. Springer (2003) muestra que un resultado común a todos los estudios analizados es que el comercio de derechos de emisión aminora el costo de los compromisos del Protocolo de Kyoto, y que la retirada de los Estados Unidos de América de dicho Protocolo tiene importantes repercusiones en su eficacia y en el sistema de comercio de emisiones que se implemente. Por otra parte, Zhang (2004) analiza la extensión del Protocolo de Kyoto a los países en desarrollo, especialmente a China, y demuestra que una amplia participación de esos países mermaría los costos de cumplimiento de los países del Anexo I.

A pesar de la abundante literatura sobre modelos aplicados a la economía del cambio climático, son pocos los estudios que se refieren ampliamente a América Latina. Medvedev y Van der Mensbrugghe (2010) intentan vincular los efectos macro a la distribución del ingreso y combinan los resultados de un modelo de equilibrio general global con un módulo climático integrado, mediante una amplia recopilación de encuestas de hogares para analizar los efectos en los países de América Latina y el Caribe. Esos autores concluyen que, con respecto a su participación en las emisiones globales, los países de la región resultan desproporcionadamente afectados por los daños derivados del cambio climático. Pese a que el bienestar disminuye en todos los hogares, aquellos dedicados a la agricultura registran algunos beneficios a raíz del incremento de los precios de los alimentos. Debido a su baja intensidad de carbono, la región puede obtener grandes beneficios de una mitigación eficaz o de un sistema de límites máximos y comercio.

En el presente estudio se analizan los posibles efectos económicos en los países en desarrollo, en particular en América Latina, de la reducción de las emisiones de $\mathrm{CO}_{2}$. Sobre la base del análisis de las interacciones entre la economía, la energía y el ambiente, se evalúan los efectos económicos y en el bienestar de la restricción de las emisiones de gases de efecto invernadero en el marco de distintos sistemas de comercio. Las simulaciones de mercados de comercio de derechos de emisión modelan las principales opciones en la mesa de las negociaciones sobre cambio climático, incluidas las que prevén contribuciones de los principales emisores en los países en desarrollo y las que suponen la participación de esos países en el comercio de derechos de emisión sin obligación de mitigar.

El análisis se concentra en dos grupos de países en desarrollo. El primero comprende a los principales actores potenciales en los mercados de comercio de derechos de emisión internacionales, como el Grupo de los 5 (G5), formado por el Brasil, China, la India, México y Sudáfrica. Debido a su contribución a las emisiones globales de $\mathrm{CO}_{2}$, estimada en más del $30 \%$ (AIE, 2010a), es importante que estos países participen en las iniciativas internacionales para reducirlas. A continuación se analizan los casos de los países de América Latina y el Caribe, incluidos el Brasil y México. Si bien su contribución a las emisiones globales de $\mathrm{CO}_{2}$ y otros gases de efecto invernadero es pequeña - menos del $6 \%$ o alrededor del $8 \%$ cuando se tienen en cuenta las emisiones relacionadas con cambios en el uso de la tierra-, la región es muy vulnerable al cambio climático (CEPAL, 2009a y 2009b).

Los representantes de los gobiernos de América Latina y el Caribe no hablan con una única voz en las negociaciones internacionales, debido a la heterogeneidad de los países de la región. Algunos, como el Estado Plurinacional de Bolivia, México y la República Bolivariana de Venezuela, son exportadores de energía, mientras que otros, como el Brasil, Chile, Costa Rica y México, son importantes actores en el mecanismo para un desarrollo limpio. Chile y México son miembros de la Organización para la Cooperación y el Desarrollo Económicos (OCDE), en tanto que el Brasil y México integran el G5. Por otra parte, los Pequeños Estados Insulares del Caribe son extremadamente vulnerables al cambio climático. En este documento se procura abordar las repercusiones económicas de diferentes hipótesis de comercio de derechos de emisión a nivel de los países de este grupo tan heterogéneo.

En la segunda sección se examinan el Protocolo de Kyoto y los mecanismos para reducir las emisiones de gases de efecto invernadero, entre ellos los mercados de carbono. En la tercera sección se presentan la metodología —incluido el modelo de equilibrio general—, la base de datos de emisiones de $\mathrm{CO}_{2}$ y las hipótesis normativas. En la cuarta sección se describen los resultados para cada conjunto de hipótesis evaluadas, en tanto que en la quinta sección se extraen las conclusiones y se discuten algunas repercusiones para las políticas de los países en desarrollo. 


\section{II \\ El Protocolo de Kyoto, el marco de modelado y las hipótesis simuladas}

El Protocolo de Kyoto se aprobó en 1997 y entró en vigencia en 2005. Su implementación se detalló en los Acuerdos de Marrakech en 2001. En conformidad con el Protocolo, se acordó que los países industrializados reducirían sus emisiones de gases de efecto invernadero en el período 2008-2012 a una media del 5,2\% con respecto a los niveles de 1990 (véase el cuadro 1) ${ }^{1}$. Según el Anexo B del Protocolo, la mayoría de los países del Anexo I deberán reducir sus emisiones, mientras que otros, en virtud de sus niveles de emisión de 1990, podrán mantener o acrecentar sus emisiones en el marco del plan de reducción.

En el Protocolo de Kyoto se establecieron tres principales mecanismos de mercado para mermar las emisiones de gases de efecto invernadero, a saber:

i) comercio internacional de derechos de emisión entre las partes participantes (países del Anexo I) en el mercado de carbono, donde los países con emisiones inferiores a sus metas pueden vender el excedente a aquellos que las han superado;

ii) aplicación conjunta, que permite a los países del Anexo I invertir en proyectos para aminorar las emisiones de gases de efecto invernadero en otros países de dicho Anexo, de modo que los créditos generados por esos proyectos valgan para sus compromisos de reducción de emisiones; y

iii) mecanismo para un desarrollo limpio (MDL), que permite a los países del Anexo I invertir en proyectos para disminuir las emisiones en países en desarrollo, de manera que los créditos generados por esos proyectos valgan para sus compromisos del Protocolo de Kyoto. En el Protocolo y en los Acuerdos de Marrakech se estableció un sistema de comercio de derechos de emisión entre 37 economías desarrolladas y en transición que representaban alrededor del $29 \%$ de las emisiones mundiales de $\mathrm{CO}_{2}$ en 2004 (WRI, 2008).

\footnotetext{
1 Las metas de reducción comprenden las emisiones de los seis principales gases de efecto invernadero: dióxido de carbono, metano, óxido nitroso, hidrofluorocarbonos, perfluorocarbonos y hexafluoruro de azufre (a estos tres últimos se los conoce como gases fluorados).
}

Con el comercio de derechos de emisión de carbono, los países que tienen excedentes de emisiones (es decir, permitidas pero no "utilizadas") pueden vender esa capacidad extra a los países que han superado sus límites. En 2005, la Unión Europea inició su sistema de comercio de derechos de emisión, que regulaba 10.000 instalaciones con un valor de 50.000 millones de dólares en el mercado de carbono internacional, o más del 75\% del mercado mundial de carbono en 2007 (Capoor y Ambrosi, 2008). Esta iniciativa sigue en curso. Al mismo tiempo, algunos sistemas nacionales de comercio de derechos de emisión están tomando forma en otros países del Anexo I, incluidos Australia, el Canadá, los Estados Unidos de América, el Japón, Nueva Zelandia y Suiza. En el caso de algunos países, como el Canadá, los Estados Unidos de América y el Japón, también existen iniciativas subregionales (Flachsland, Marschinski y Edenhofer, 2009).

Aunque la aplicación de impuestos sobre las emisiones de carbono es relativamente nueva en los países en desarrollo, estos se están implementando en muchos de ellos - ya sea con sistemas de comercio de derechos de emisión o sin ellos-como un instrumento independiente o combinado con otros instrumentos de fijación de precios del carbono, por ejemplo, el impuesto a la energía (OCDE, 2013). Asimismo, en muchos de ellos se están estimando los cobeneficios locales de la mitigación de las emisiones de $\mathrm{CO}_{2}$, mientras que se estudian los sistemas de límites máximos y comercio, subastas y otras políticas de precios para actividades específicas (CEPAL, 2009a; Johnson y otros, 2009; AIE, 2010b). El valor del impuesto sobre las emisiones de carbono o su equivalente y los cobeneficios dependen del sistema, las actividades involucradas, la cobertura geográfica y el año, entre otros factores.

Sin embargo, estos mercados regionales o nacionales son limitados en la medida en que pueden excluir a algunos países particularmente eficaces en la mitigación de las emisiones de gases de efecto invernadero, entre ellos algunos países en desarrollo, o perder los beneficios de la flexibilidad de mercados más amplios y profundos. Evans (2003) sostiene que el comercio internacional de derechos de emisión tiene 
el potencial para disminuir el costo de la reducción de las emisiones y promover inversiones respetuosas del ambiente en las economías en transición. De la Torre, Fajnzylber y Nash (2009) van más allá de las economías en transición y afirman que una solución global y rentable solo se logrará mediante la participación de los países que pueden reducir las emisiones de gases de efecto invernadero a bajo costo.

CUADRO 1

Partes del Protocolo de Kyoto: niveles de emisión en el año de referencia y limitaciones de emisión

\begin{tabular}{|c|c|c|c|}
\hline Parte & $\begin{array}{l}\text { Compromiso de limitación o } \\
\text { reducción de las emisiones } \\
\text { (en porcentaje del nivel del año } \\
\text { o período de referencia })^{\mathrm{a}, \mathrm{b}}\end{array}$ & $\begin{array}{l}\text { Año de referencia } \\
\text { para los gases } F\end{array}$ & $\begin{array}{c}\text { Emisiones del país en el año de } \\
\text { referencia (en toneladas de CO2 } \\
\text { equivalentes) }{ }^{\mathrm{c}}\end{array}$ \\
\hline Australia & 108 & 1990 & \\
\hline Austria & 87 & 1990 & 79049657 \\
\hline Belarús ${ }^{d}$ & $92^{\mathrm{e}}$ & 1995 & \\
\hline Bélgica & 92,5 & 1995 & 145728763 \\
\hline Bulgaria $^{\mathrm{d}}$ & 92 & 1995 & 132618658 \\
\hline Canadá & 94 & 1990 & 593998462 \\
\hline Croacia $^{\mathrm{d}}$ & 95 & & \\
\hline República Checa ${ }^{\mathrm{d}}$ & 92 & 1995 & 194248218 \\
\hline Dinamarca & 79 & 1995 & 69978070 \\
\hline Estonia $^{\mathrm{d}}$ & 92 & 1995 & 42622312 \\
\hline Unión Europea & 92 & 1990 о 1995 & 4265517719 \\
\hline Finlandia & 100 & 1995 & 71003509 \\
\hline Francia & 100 & 1990 & 563925328 \\
\hline Alemania & 79 & 1995 & 1232429543 \\
\hline Grecia & 125 & 1995 & 106987169 \\
\hline Hungría $^{d}$ & 94 & 1995 & 115397149 \\
\hline Islandia & 110 & 1990 & 3367972 \\
\hline Irlanda & 113 & 1995 & 55607836 \\
\hline Italia & 93,5 & 1990 & 516850887 \\
\hline Japón & 94 & 1995 & 1261331418 \\
\hline Letonia $^{\mathrm{d}}$ & 92 & 1995 & 25909159 \\
\hline Liechtenstein & 92 & 1990 & 229483 \\
\hline Lituania $^{\mathrm{d}}$ & 92 & 1995 & 49414386 \\
\hline Luxemburgo & 72 & 1995 & 13167499 \\
\hline Mónaco & 92 & 1995 & 107658 \\
\hline Países Bajos & 94 & 1995 & 213034498 \\
\hline Nueva Zelandia & 100 & 1990 & 61912947 \\
\hline Noruega & 101 & 1990 & 49619168 \\
\hline Polonia $\mathrm{a}^{\mathrm{d}}$ & 94 & 1995 & 563442774 \\
\hline Portugal & 127 & 1995 & 60147642 \\
\hline Rumania $^{\mathrm{d}}$ & 92 & 1989 & 278225022 \\
\hline Federación de Rusia ${ }^{\mathrm{d}}$ & 100 & 1995 & 3323419064 \\
\hline Eslovaquia $^{\mathrm{d}}$ & 92 & 1990 & 72050764 \\
\hline Eslovenia $^{\mathrm{d}}$ & 92 & 1995 & 20354042 \\
\hline España & 115 & 1995 & 289773205 \\
\hline Suecia & 104 & 1995 & 72151646 \\
\hline Suiza & 92 & 1990 & 52790957 \\
\hline Ucrania $^{\mathrm{d}}$ & 100 & 1990 & 920836933 \\
\hline $\begin{array}{l}\text { Reino Unido de Gran Bretaña } \\
\text { e Irlanda del Norte }\end{array}$ & 87,5 & 1995 & 779904144 \\
\hline
\end{tabular}

Fuente: Convención Marco de las Naciones Unidas sobre el Cambio Climático (CMNucc) [en línea] http://unfccc.int/portal_espanol/ items/3093.php.

a Las metas establecidas en el acuerdo de distribución de las cargas de la Unión Europea (15) se muestran en cursiva.

b Las partes del Anexo I con un año de referencia distinto de 1990 son: Bulgaria (1988), Eslovenia (1986), Hungría (promedio de 1985-1987), Polonia (1988) y Rumania (1989).

c Los datos relativos al año de referencia son los establecidos durante el proceso de revisión inicial.

d Países en proceso de transición hacia una economía de mercado.

e La enmienda del Protocolo de Kyoto en que se fija una meta de reducción de emisiones para Belarús todavía no ha entrado en vigencia. Nota: los gases F son los gases fluorados: hidrofluorocarbonos, perfluorocarbonos y hexafluoruro de azufre. 


\section{III}

\section{Modelo económico para el cambio climático y el comercio de derechos de emisión: el modelo GTAP-E}

Desde la firma del Protocolo de Kyoto, se ha ampliado la literatura económica dedicada al modelado de su implementación y al comercio de derechos de emisión de carbono. Springer (2003) recopiló los resultados de 25 modelos del mercado de permisos de emisión de gases de efecto invernadero comercializables en el marco del Protocolo de Kyoto. Dichos modelos se categorizan en los siguientes cinco grupos principales no exclusivos (véase el gráfico 1):

i) modelos de evaluación integrados, que incluyen procesos físicos y sociales y un componente económico representado por uno de los siguientes modelos:

ii) modelos de equilibrio general computable;

iii) modelos de comercio de derechos de emisión; iv) modelos macroeconómicos neokeynesianos, y

v) modelos de sistemas de energía

Los modelos de equilibrio general y los modelos macroeconómicos neokeynesianos se caracterizan como descendentes, pues utilizan datos económicos agregados sobre todos los sectores de la economía. Por otra parte, los modelos de sistemas de energía ofrecen un mayor detalle sectorial para el sector de energía que los modelos de equilibrio general computable y los modelos macroeconómicos, y en consecuencia se los denomina modelos ascendentes. En este estudio se utilizó un modelo de equilibrio general aplicado, el GTAP-E, una versión modificada del Proyecto de Análisis del Comercio Global (GTAP) y la base de datos asociada . El modelo GTAP-E (Burniaux y Truong, 2002;

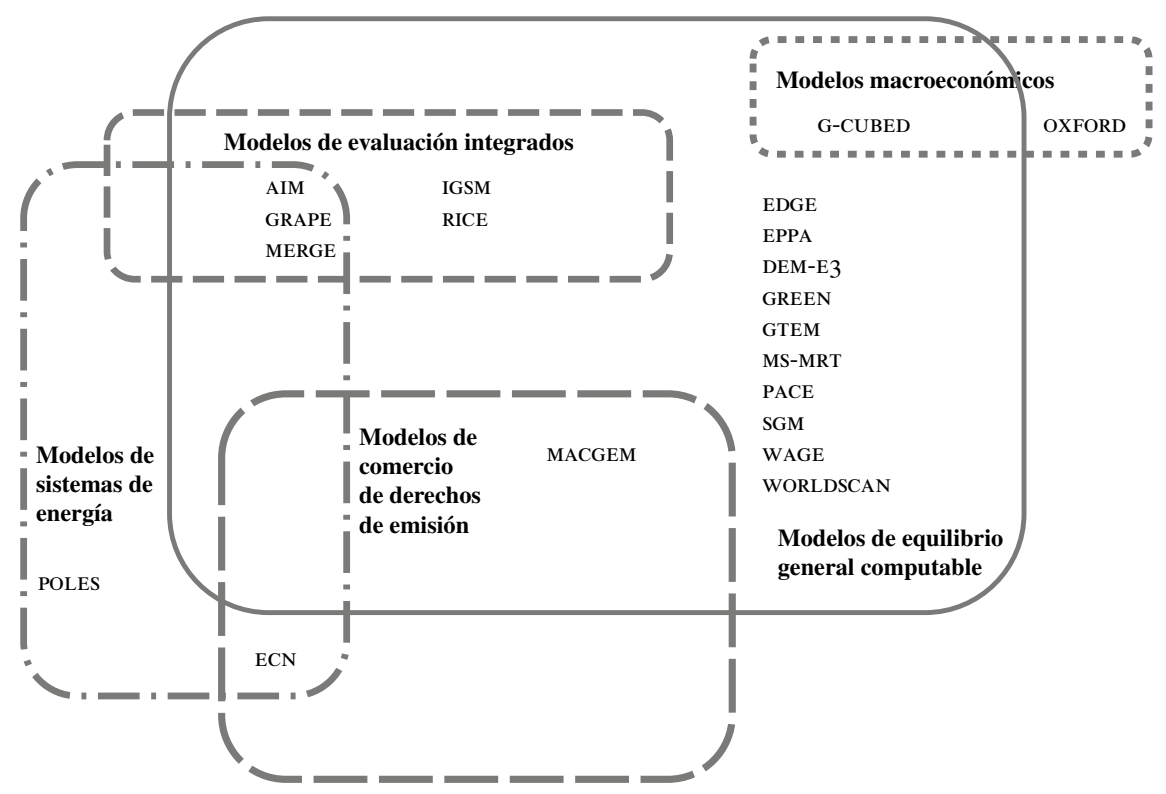

Fuente: U. Springer, "The market for tradable GHG permits under the Kyoto Protocol: A survey of model studies", Energy Economics, vol. 25, $\mathrm{N}^{\circ}$ 5, Amsterdam, Elsevier, 2003.

Nota: el modelo GTAP-E se clasifica como un modelo de equilibrio general computable. 
McDougall y Golub, 2009) es una extensión del modelo GTAP (Hertel, 1997; Tsigas, Frisvold y Kuhn, 1997), que es un modelo de equilibrio general aplicado estándar, estático, multirregional y multisectorial, que incluye el tratamiento explícito de los márgenes de comercio internacional y transporte, el ahorro y la inversión a nivel mundial, y la respuesta de precios e ingresos en los países. Supone competencia perfecta, rendimientos de escala constantes y la especificación de Armington para los flujos comerciales bilaterales, que diferencia el comercio según el origen ${ }^{2}$. El modelo GTAP-E se utilizó para analizar el comercio de derechos de emisión en Hamasaki y Truong (2001); Hamasaki (2004); Nijkamp, Wang y Kremers (2005); Dagoumas, Papagiannis y Dokopoulos (2006), y Houba y Kremers (2007).

El modelo GTAP-E incorpora un tratamiento modificado de la demanda de energía, que incluye la sustitución entre energía y capital y entre combustibles, la contabilidad de $\mathrm{CO}_{2}$ y la tributación y el comercio de derechos de emisión. Constituye un enfoque de modelado de energía descendente que, dada una descripción económica detallada a nivel macro, estima la demanda de insumos de energía en términos de demanda de

${ }^{2} \mathrm{Al}$ igual que los demás, los modelos de equilibrio general computable presentan algunas limitaciones, como son su dependencia de una considerable cantidad de datos estadísticos y de parámetros y elasticidades de calidad (estimados fuera del modelo), su escasa representación del comportamiento de la inversión y las reglas de cierre elegidas para las simulaciones (O'Ryan, De Miguel y Miller, 2000; Schuschny, Durán y De Miguel, 2007). producción sectorial. Esos dos tipos de demanda son estimados a partir de funciones de costo o producción agregada $^{3}$.

Desde el punto de vista de la producción, el modelo GTAP-E perfecciona el modelo GTAP estándar mediante un nuevo sistema de producción con niveles intermedios adicionales de estratificación. En este sistema la energía se incorpora en el estrato de valor agregado (véase el gráfico 2), de modo que los insumos de energía se combinan con el capital para producir un compuesto energía-capital, que a su vez se combina con otros insumos primarios en un estrato de valor agregado-energía mediante una función de elasticidad de sustitución constante. Los productos básicos del sector de energía se separan también en electricidad y productos distintos de la electricidad (véase el gráfico 3), con un nivel de sustitución dentro del grupo de productos distintos de la electricidad (ONELY) y entre los grupos de productos de electricidad y distintos de la electricidad

\footnotetext{
${ }^{3}$ Estas capacidades ofrecen flexibilidad en las opciones de reducción de las emisiones después de la aplicación de impuestos sobre las emisiones de carbono, cuotas o el comercio de los derechos de emisión, pues los países y sectores no se ven limitados a alcanzar sus metas reduciendo el producto interno bruto (PIB), sino que pueden buscar una nueva solución óptima en su estructura de producción, su combinación capital-energía y sus patrones de consumo. Esto puede suponer opciones de eficiencia energética y cambios en la composición del valor agregado (incluida la energía) por la vía de las elasticidades de sustitución valor agregado-energía y capital-energía. El modelo no permite modificaciones a los coeficientes técnicos entre insumos o a la relación entre valor agregado e insumos.
}

GRÁFICO 2

\section{Estructura de producción del modelo GTAP-E}
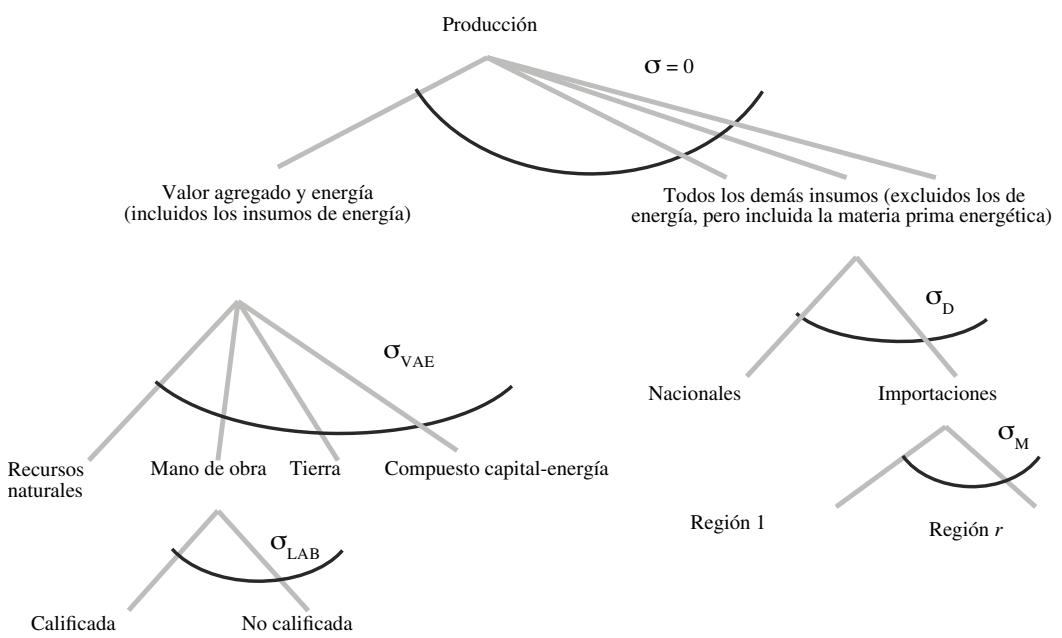

nergía, pero incluida la materia prima energética)

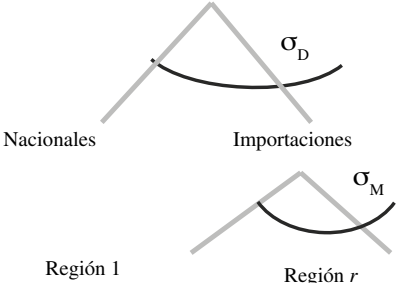

Región 1

Región $r$

Fuente: J.M. Burniaux y T.P. Truong, “GTAP-E: An energy-environmental version of the GTAP model”, GTAP Technical Paper, ํㅜ 16, West Lafayette, Center for Global Trade Analysis, Purdue University, 2002. 


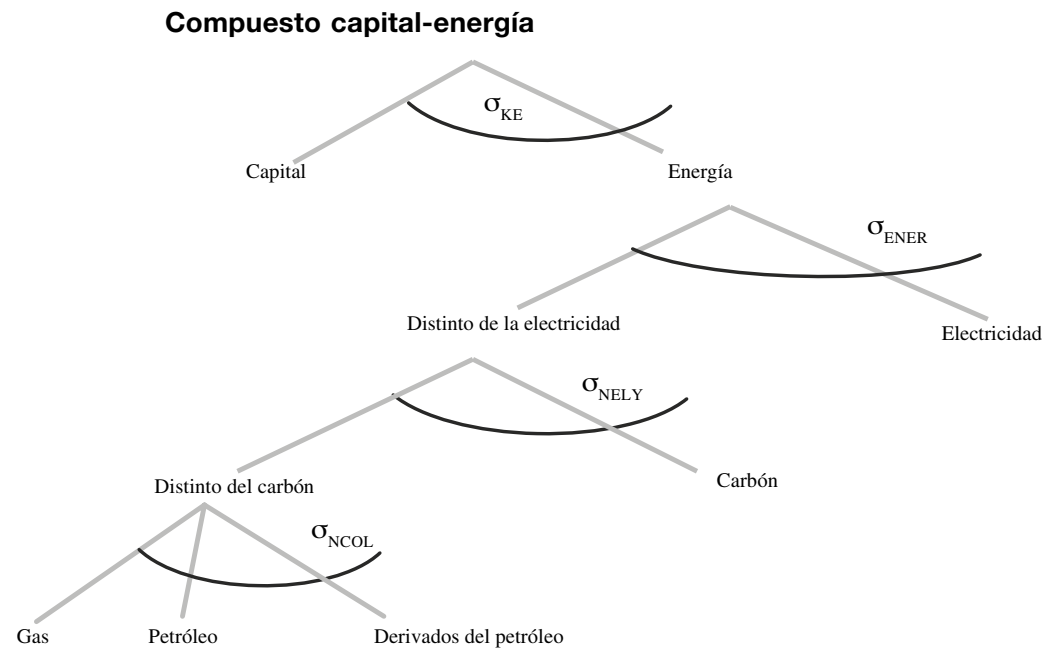

Fuente: J.M. Burniaux y T.P. Truong, “GTAP-E: An energy-environmental version of the GTAP model”, GTAP Technical Paper, N 16, West Lafayette, Center for Global Trade Analysis, Purdue University, 2002.

$(\sigma E N E R)$. Esta estratificación continúa con la separación de los productos distintos de la electricidad en carbón y distintos del carbón, y de estos últimos en gas, petróleo y derivados del petróleo, con una elasticidad de sustitución de $\sigma N C O L^{4}$.

El modelo GTAP-E también modifica el consumo privado y del gobierno (véanse los gráficos 4 y 5 ), separando los productos básicos de energía de los otros. Con respecto al consumo del gobierno, las elasticidades de sustitución $(\sigma \mathrm{GENNE}=0,5$ y $\sigma \mathrm{GEN}=1)$ permiten la sustitución entre productos básicos de energía y otros. Sin embargo, si $\sigma \mathrm{GENNE}=\sigma \mathrm{GEN}=1$, la estructura del GTAP-E vuelve al modelo GTAP estándar. El consumo de los hogares sigue el modelo GTAP estándar, que utiliza la forma funcional de diferencia constante de elasticidades. El modelo GTAP-E especifica el compuesto de energía empleando una forma funcional de elasticidad de sustitución constante con una elasticidad de sustitución de OPEN $=1$.

En el presente estudio se utiliza una nueva versión del modelo GTAP-E (McDougall y Golub, 2009) que modifica la versión anterior (Burniaux y Truong, 2002) mediante:

i) el restablecimiento del comercio de derechos de emisión con bloques comerciales;

ii) el cálculo ascendente de las emisiones de dióxido de carbono;

\footnotetext{
${ }^{4}$ Esta estructura de producción puede modificarse incluso más para incluir la producción de biocombustible, como en Birur, Hertel y Tyner (2007).
}

iii) el restablecimiento de los impuestos sobre las emisiones de carbono, sin convertir las tasas de específicas a ad valorem;

iv) la reorganización de la estructura productiva para agrupar las ecuaciones por estrato y con un conjunto completo de variables de cambio tecnológico, y

v) la revisión del cálculo del aporte de los ingresos netos del comercio de permisos a los cambios en el bienestar.

En este caso, el modelo GTAP-E incluye permisos de emisión y comercio de derechos de emisión al prever la negociación de permisos entre los miembros de los bloques comerciales. Esto posibilita que las emisiones y sus cuotas sean las mismas a nivel del bloque. El modelo también hace posible la tributación de las emisiones de carbono, relacionando su nivel con una tasa impositiva.

\section{Datos económicos, emisiones de $\mathrm{CO}_{2}$ y parámetros}

En el modelo GTAP-E se modifica la base de datos GTAP estándar al incluir las emisiones de $\mathrm{CO}_{2}$ por región, producto básico y uso. En este trabajo se utiliza la versión 6 de la base de datos GTAP, que abarca 87 regiones y cuyo año de referencia es $2001^{5}$. Los datos relativos

\footnotetext{
5 Se intentó utilizar la versión 7 convirtiendo los datos sobre las emisiones de $\mathrm{CO}_{2}$ elaborados por Lee (2008) a un formato compatible con el modelo GTAP. Si bien Lee elaboró datos sobre las emisiones de $\mathrm{CO}_{2}$ para la versión 7.0 de la base de datos GTAP (113 regiones, año de referencia 2004), a diferencia de los datos de las emisiones de $\mathrm{CO}_{2}$ para la versión 6.0, estos no incluían la distinción entre fuentes nacionales e importadas.
} 
GRÁFICO 4

\section{Consumo del gobierno del modelo GTAP-E}

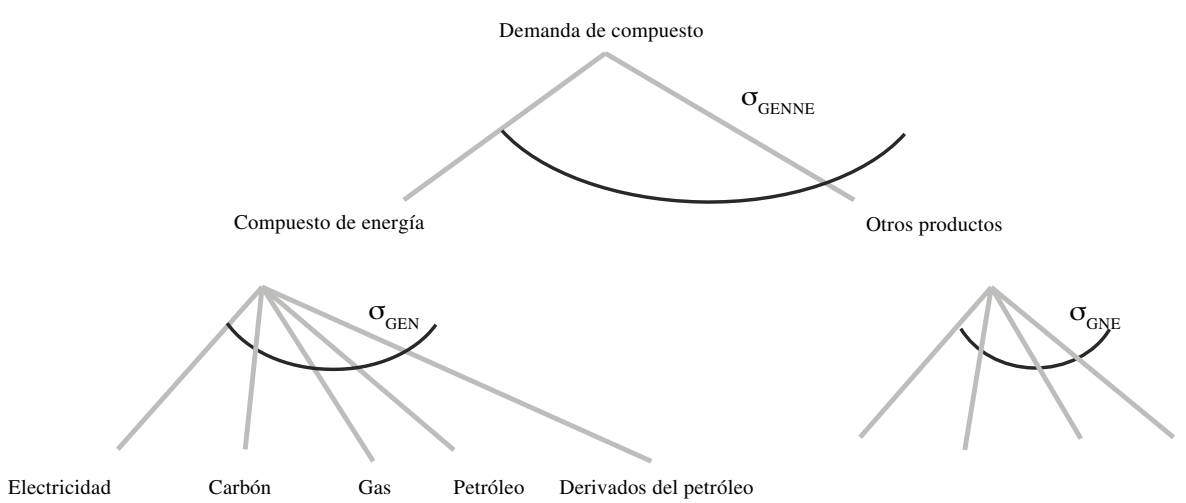

Fuente: J.M. Burniaux y T.P. Truong, "GTAP-E: An energy-environmental version of the GTAP model”, GTAP Technical Paper, N 16, West Lafayette, Center for Global Trade Analysis, Purdue University, 2002.

GRÁFICO 5

\section{Consumo de los hogares del modelo GTAP-E}

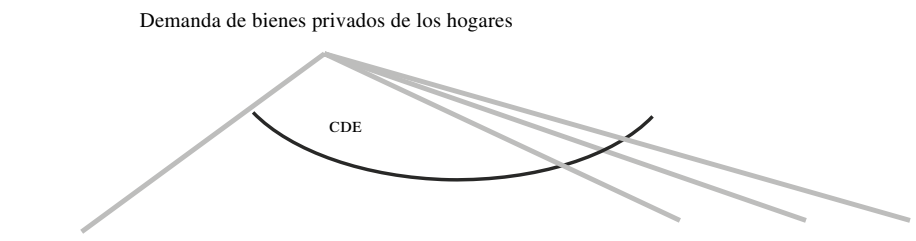

Compuesto de energía

Otros productos

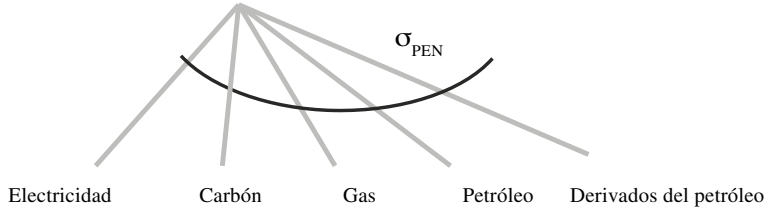

Fuente: J.M. Burniaux y T.P. Truong, “GTAP-E: An energy-environmental version of the GTAP model”, GTAP Technical Paper, $\mathrm{N}^{\circ} 16$, West Lafayette, Center for Global Trade Analysis, Purdue University, 2002.

a las emisiones de $\mathrm{CO}_{2}$ se tomaron de Lee (2008) y se convirtieron a un formato compatible con el modelo GTAP (Ludeña, 2007). Esos datos comprenden las emisiones derivadas del uso intermedio y del consumo del gobierno y los hogares de productos nacionales e importados. Este trabajo constituye un avance con respecto a los estudios anteriores basados en el modelo GTAP-E, pues en la nueva versión empleada se corrigen algunos defectos que aparecen en Burniaux y Truong (2002) y se mejoran los datos económicos, así como los relativos a las emisiones de $\mathrm{CO}_{2}$.

Con respecto a los parámetros, el modelo GTAP-E incluye elasticidades de sustitución para capitalsubproducción de energía (OKE), subproducción de energía (OENER), subproducción de energía distinta de la electricidad (ONELY) y subproducción de energía distinta del carbón (ONCOL). También modifica la elasticidad de sustitución para factores primarios ( $\sigma \mathrm{VAE})$, pues añade una dimensión regional a este parámetro del GTAP. En el presente estudio se utilizan parámetros de sustitución estimados econométricamente por Beckman y Hertel (2009).

La base de datos GTAP se divide en 19 sectores y 25 regiones (véanse los cuadros 2 y 3 ), con especial atención a los países en desarrollo, incluidos los de América Latina y el Caribe. Las agrupaciones sectoriales se concentran en los sectores de energía e intensivos en energía, así como en los sectores relacionados con las emisiones de carbono, como pulpa y papel, productos químicos, productos minerales (producción de hormigón) y productos de metal. 
Agrupaciones sectoriales para todos los países de la base de datos GTAP, versión 6

\begin{tabular}{|c|c|c|}
\hline $\mathrm{N}^{\mathrm{o}}$ & Sector & Descripción (57 productos básicos) \\
\hline 1 & Cultivos & $\begin{array}{l}\text { Arroz con cáscara, trigo, granos de cereales, frutas y verduras, semillas oleaginosas, cultivos } \\
\text { de azúcar, fibras de origen vegetal, otros cultivos }\end{array}$ \\
\hline 2 & Ganadería & Ganado, cerdos, aves de corral, leche cruda, lana \\
\hline 3 & Silvicultura & Silvicultura \\
\hline 4 & Pesca & Pesca \\
\hline 5 & Carbón & Extracción de carbón \\
\hline 6 & Crudo & Extracción de petróleo \\
\hline 7 & Gas & Extracción y distribución de gas \\
\hline 8 & Minería & Minería \\
\hline 9 & Industria ligera & $\begin{array}{l}\text { Alimentos procesados (carne, aceites y grasas vegetales, productos lácteos, arroz procesado } \\
\text { y azúcar, entre otros), bebidas y tabaco, textiles, prendas de vestir, productos de cuero, } \\
\text { productos de madera }\end{array}$ \\
\hline 10 & Papel & Productos de papel \\
\hline 11 & Productos petrolíferos procesados & Productos derivados del petróleo y del carbón \\
\hline 12 & Productos químicos & Productos químicos, de caucho y de plástico \\
\hline 13 & Productos minerales & Vidrio, hormigón y otros productos minerales \\
\hline 14 & Productos de metal & Metales ferrosos y otros \\
\hline 15 & Industria pesada & $\begin{array}{l}\text { Productos de metal, vehículos motorizados y sus partes, equipos de transporte, maquinaria } \\
\text { y equipos, otras manufacturas }\end{array}$ \\
\hline 16 & Electricidad & Electricidad \\
\hline 17 & Construcción & Construcción \\
\hline 18 & Transporte & Servicios de transporte terrestre, servicios de transporte aéreo y acuático \\
\hline 19 & Otros servicios & $\begin{array}{l}\text { Comunicación, servicios financieros, seguros, servicios comerciales, servicios recreativos y } \\
\text { de otro tipo, administración pública, viviendas }\end{array}$ \\
\hline
\end{tabular}

Fuente: elaboración propia sobre la base de información de GTAP Data Base.

CUADRO 3

Agrupaciones regionales de la base de datos GTAP, versión 6

\begin{tabular}{|c|c|c|}
\hline $\mathrm{N}^{\mathrm{o}}$ & Región/país & Descripción (87 países) \\
\hline 1 & Estados Unidos de América & Estados Unidos de América \\
\hline 2 & Unión Europea-15 países & $\begin{array}{l}\text { Alemania, Austria, Bélgica, Dinamarca, España, Finlandia, Francia, Grecia, Irlanda, Italia, } \\
\text { Luxemburgo, Países Bajos, Portugal, Reino Unido de Gran Bretaña e Irlanda del Norte, } \\
\text { Suecia }\end{array}$ \\
\hline 3 & Japón & Japón \\
\hline 4 & Unión Europea-12 países & $\begin{array}{l}\text { Bulgaria, Chipre, Eslovaquia, Eslovenia, Estonia, Hungría, Letonia, Lituania, Malta, } \\
\text { Polonia, República Checa, Rumania }\end{array}$ \\
\hline 5 & Otros europeos Anexo I & Croacia, Federación de Rusia, resto de la ex Unión Soviética \\
\hline 6 & Resto de Anexo I & $\begin{array}{l}\text { Australia, Canadá, Noruega, Nueva Zelandia, Suiza, resto de la Asociación Europea de } \\
\text { Libre Comercio (AELC) }\end{array}$ \\
\hline 7 & Resto de Europa & Albania, resto de Europa oriental, resto de Europa \\
\hline 8 & China & China \\
\hline 9 & India & India \\
\hline 10 & Sudáfrica & Sudáfrica \\
\hline 11 & Exportadores de energía & $\begin{array}{l}\text { Indonesia, Malasia, Viet Nam, resto de Asia sudoriental, resto de Asia occidental, } \\
\text { resto de África septentrional, África central, centro-sur de África, resto de África oriental }\end{array}$ \\
\hline 12 & Argentina & Argentina \\
\hline 14 & Bolivia (Estado Plurinacional de) & Estado Plurinacional de Bolivia \\
\hline 13 & Brasil & Brasil \\
\hline 15 & Chile & Chile \\
\hline 16 & Colombia & Colombia \\
\hline 17 & Ecuador & Ecuador \\
\hline 18 & México & México \\
\hline 19 & Paraguay & Paraguay \\
\hline 20 & Perú & Perú \\
\hline 21 & Uruguay & Uruguay \\
\hline 22 & Venezuela (República Bolivariana de) & República Bolivariana de Venezuela \\
\hline 23 & Centroamérica & Belice, Costa Rica, El Salvador, Guatemala, Honduras, Nicaragua, Panamá \\
\hline 24 & Caribe & Cuba, Haití, Jamaica, Puerto Rico, República Dominicana, Trinidad y Tabago, otros. \\
\hline 25 & Resto del mundo & Resto del mundo \\
\hline
\end{tabular}

Fuente: elaboración propia sobre la base de información de GTAP Data Base. 


\section{Escenarios de políticas}

Flachsland, Marschinski y Edenhofer (2009) analizan el comercio internacional de derechos de emisión en el contexto de lo que denominan "estructuras de negociación", con dos opciones descendentes (impulsadas por la CMNUCC) y tres ascendentes (impulsadas por regiones o países individuales). Estos dos enfoques tienen contrapartidas en términos de la viabilidad política, la eficacia del sistema de comercio para reducir las emisiones de gases de efecto invernadero y los costos. A continuación se procura cubrir estas diferentes "estructuras de negociación" mediante la formulación de varias hipótesis de reducción y comercio de las emisiones de dióxido de carbono, con la participación de los países en desarrollo y sin ella.

Con el GTAP-E se modela el comercio de derechos de emisión mediante la división del mundo en bloques comerciales, cuyos miembros negocian los permisos de emisión entre sí. Esto permite formular hipótesis en las que no hay comercio de derechos de emisión y cada región constituye su propio bloque. En la hipótesis de comercio del Anexo I, solo los países incluidos en esa categoría forman un bloque comercial que excluye a las demás regiones. En la hipótesis de comercio mundial, todas las regiones negocian permisos de emisión de carbono y el mundo se convierte en un único bloque comercial. Sobre esta base se formulan las siguientes cuatro hipótesis principales:

- Protocolo de Kyoto sin comercio de derechos de emisión (Kyontr);

- Protocolo de Kyoto con comercio de derechos de emisión entre los países del Anexo I (Kyotr);

- Protocolo de Kyoto con comercio de derechos de emisión entre los países del Anexo I y participación de algunos países en desarrollo (Kyotr3 y Kyotrla);
- Protocolo de Kyoto con comercio mundial de derechos de emisión (Kyowtr).

En la primera hipótesis (base), cada país del Anexo I debe cumplir en forma individual con sus metas de reducción de las emisiones de $\mathrm{CO}_{2}$ establecidas en Kyoto sin comercio de derechos de emisión entre países. En este caso, los países del Anexo I cumplen con sus compromisos individualmente, sin contar con mecanismos de flexibilidad. Las limitaciones de emisiones de $\mathrm{CO}_{2}$ empleadas en este estudio se detallan en el cuadro 1. Si bien los Estados Unidos de América no han ratificado el Protocolo de Kyoto, para fines comparativos se supone una meta de reducción del $7 \%$ para ese país.

A objeto de conciliar el calendario del Protocolo de Kyoto con el año de referencia de la base de datos GTAP-E, se asumió que los países del Anexo I reducirían sus emisiones de carbono entre 1990 y 2008-2012, el primer período de compromiso del Protocolo, y se tuvieron en cuenta los niveles de emisión de $\mathrm{CO}_{2}$ de 2001 (año de referencia de los datos relativos al $\mathrm{CO}_{2}$ empleados en este estudio). Para ello, se utilizaron las emisiones de $\mathrm{CO}_{2}$ antropogénicas agregadas de 1990 y 2000 (CMNUCC, 2007). A partir de la tasa de variación anual media de las emisiones entre 1990 y 2000, se extrapolaron los datos del año 2000 para estimar los niveles de emisión de 2001. Con estos niveles, se ajustaron las metas de reducción de emisiones basadas en las cifras de 1990 al año 2001 mediante la comparación de los niveles de emisión establecidos como metas con los obtenidos con respecto a 2001. Las limitaciones de emisión estimadas son las siguientes: poco menos de un $21 \%$ para los Estados Unidos de América, casi un 6\% para la Unión Europea de 15 miembros, bordeando un 12\% para el Japón y algo menos de un $16 \%$ para el resto de la categoría del Anexo I (véase el cuadro 4).

CUADRO 4

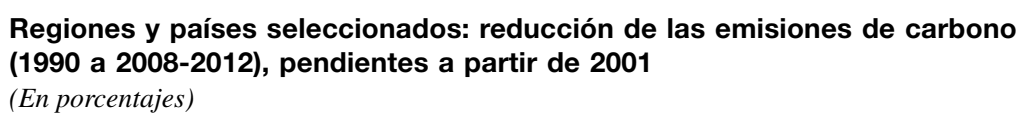

\begin{tabular}{llc}
\hline País/región & \multicolumn{1}{c}{ Descripción } & Variación en las emisiones de $\mathrm{CO}_{2}$ \\
\hline Estados Unidos de América & Estados Unidos de América & 20,78 \\
Unión Europea-15 países & Unión Europea de 15 miembros & $-5,37$ \\
Japón & Japón & $-11,8$ \\
Unión Europea-12 países & Unión Europea (nuevos miembros) & 48,81 \\
Otros europeos Anexo I & Otros países europeos del Anexo I & 64,31 \\
Resto de Anexo I & Resto de países del Anexo I & $-15,89$ \\
Resto de Europa & Resto de Europa & 48,81
\end{tabular}

Fuente: elaboración propia sobre la base de Convención Marco de las Naciones Unidas sobre el Cambio Climático (CMNUCC), "National greenhouse gas inventory data for the period 1990-2005" (FCCC/SBI/2007/30), 2007 [en línea] http://unfccc.int/resource/docs/2007/sbi/ eng/30.pdf. 
En el marco de la primera hipótesis se probó también la situación en que algunos países en desarrollo, a saber el G5 (Brasil, China, India, México y Sudáfrica), reducen sus emisiones un 5\%. La elección de esos países se debe a que probablemente contribuirían a la disminución de las emisiones en las negociaciones sobre cambio climático. Las cifras correspondientes a la reducción de las emisiones son arbitrarias, pero pueden dar una medida del impacto de reducción potencial de esos países ${ }^{6}$.

En la segunda hipótesis se asume la reducción de las emisiones solo por los países del Anexo I y la negociación de derechos de emisión exclusivamente entre ellos. Las limitaciones de emisión aplicadas a los países del Anexo I son las mismas de la primera hipótesis, a las que se suma el excedente de créditos de emisiones (conocido como "aire caliente") de la ex Unión Soviética7. El "aire caliente" representa la cantidad de emisiones reducidas que supera los requisitos de emisiones previstos de acuerdo con el Protocolo de Kyoto, incluso en ausencia de limitaciones. Se asume que las emisiones de $\mathrm{CO}_{2}$ de los 12 nuevos miembros de la Unión Europea (UE12) y de la categoría Otros países europeos del Anexo I se mantienen igual (meta de reducción de las emisiones igual a cero), pues esos niveles les permiten emitir un $49 \%$ y un $64 \%$ más, respectivamente, de lo permitido en el marco del Protocolo (véase el cuadro 4). Con respecto al "aire caliente" de los países de Europa oriental y la ex Unión Soviética, se estudian varias hipótesis con esta variable y sin ella ${ }^{8}$.

En la tercera hipótesis se tiene en cuenta la participación de los países que no figuran en el Anexo I. En primer lugar, se asume el comercio de derechos de emisión entre los países del Anexo I y los principales emisores entre los países en desarrollo, que incluyen el Brasil, China, la India, México y Sudáfrica (G5). $\mathrm{Al}$ igual que en la primera hipótesis, las emisiones de este grupo se reducen un 5\%. El análisis se concentra a continuación en los países latinoamericanos y caribeños y en su potencial para participar en el comercio de

\footnotetext{
${ }^{6}$ Anger (2008) también estudia la hipótesis en que los derechos de emisión extra no se asignan a instalaciones en la ex Unión Soviética, pues pone en duda que esa estrategia prevalezca en el futuro.

${ }^{7}$ El excedente de emisiones originado en la recesión económica en la ex Unión Soviética (al que a menudo se hace referencia como "aire caliente") es suficiente para compensar las reducciones requeridas en los demás países del Anexo I.

${ }^{8}$ De utilizarse el comercio de derechos de emisión, el excedente de emisiones de la ex Unión Soviética puede, en principio, transferirse a otras partes del Anexo I sin costo alguno.
}

derechos de emisión, ya sea suponiendo reducciones por parte de los Estados Unidos de América o sin ellas. En este caso no se asumen cuotas de reducción específicas para esos países y sus emisiones se mantienen igual (no aumentan ni disminuyen).

En el cuarto marco hipotético se presume un verdadero sistema de límites máximos y comercio de derechos de emisión entre los países del Anexo I y los demás. Se formulan dos escenarios. En el primero, solo los países del Anexo I reducen sus emisiones y se incluye el "aire caliente" de los países de la ex Unión Soviética. La segunda hipótesis ofrece una visión alternativa en que los países del Anexo I y del G5 reducen sus emisiones, pero no se incluye el "aire caliente". En ambos casos, las limitaciones de las cuotas de emisión de $\mathrm{CO}_{2}$ para todos los demás países, incluidos los países en desarrollo, se fijan en cero.

Por último, dentro de cada uno de los cuatro principales marcos hipotéticos, se examinaron los distintos casos según se redujeran o no las emisiones de los Estados Unidos de América. La participación de ese país en el mercado de carbono, en las situaciones en que está previsto, está condicionada a la reducción de sus propias emisiones (si las reduce participa, de lo contrario no lo hace).

En las hipótesis que prevén el comercio de derechos de emisión, los países que participaban en dicho comercio formaban parte de un bloque comercial. En la tercera hipótesis, en que los países que no figuran en el Anexo I también participan en el comercio de derechos de emisión, se modificó el archivo de parámetros y cierre del GTAP-E para permitir las negociaciones entre los países del Anexo I y regiones específicas. Como mencionan McDougall y Golub (2009), en el cierre estándar sin comercio de derechos de emisión, las emisiones son siempre iguales a la cuota. En otras palabras, la cuota de emisiones carece de sentido y refleja las emisiones como si no se hubiera impuesto alguna restricción. Sin embargo, cuando se permite el comercio, las emisiones y las cuotas regionales se disocian porque las emisiones reales se vuelven exógenas y las cuotas de emisiones, endógenas.

Las hipótesis descritas se resumen en el cuadro 5. En la columna "Estados Unidos de América" se indica si hay reducción de las emisiones de $\mathrm{CO}_{2}$ en ese país. La participación de los Estados Unidos de América en el comercio de derechos de emisión —entre los países del Anexo I en las hipótesis que lo contemplanestá supeditada a la reducción de sus emisiones. En 
la columna "Ex Unión Soviética" se muestran las hipótesis que incluyen el "aire caliente" de los países de esa categoría. En la columna "Grupo de los 5" se indican las hipótesis en que el Brasil, China, la India, México y Sudáfrica reducen sus emisiones un 5\%. Estos escenarios de política cubren las estructuras de negociación de derechos de emisión descritas por Flachsland, Marschinski y Edenhofer (2009), con una combinación de enfoques descendentes y ascendentes, es decir, iniciativas globales combinadas con sistemas de comercio nacionales o regionales ${ }^{9}$.

${ }^{9}$ En estas hipótesis se supone un único precio válido para todos los bloques comerciales o países, sin imperfecciones de mercado, como la monopolización de los mercados, y con divulgación total de precios entre los países participantes.

CUADRO 5

Escenarios de política para el comercio de derechos de emisión

\begin{tabular}{|c|c|c|c|c|c|}
\hline $\mathrm{N}^{\mathrm{o}}$ & Hipótesis & Descripción & $\begin{array}{l}\text { Estados } \\
\text { Unidos de } \\
\text { América }\end{array}$ & $\begin{array}{l}\text { Ex Unión } \\
\text { Soviética }\end{array}$ & $\begin{array}{l}\text { Grupo de } \\
\quad \operatorname{los} 5\end{array}$ \\
\hline 1 & Kyontrla & Kyoto sin comercio de derechos de emisión, con Estados Unidos & $\checkmark$ & & \\
\hline 2 & Kyontr1b & Kyoto sin comercio de derechos de emisión, sin Estados Unidos & & & \\
\hline 3 & Kyontr2a & Kyoto sin comercio de derechos de emisión, con Estados Unidos y G5 (-5\%) & $\checkmark$ & & $\checkmark$ \\
\hline 4 & Kyontr $2 b$ & $\begin{array}{l}\text { Kyoto sin comercio de derechos de emisión, sin Estados Unidos pero con G5 } \\
(-5 \%)\end{array}$ & & & $\checkmark$ \\
\hline 5 & Kyotr0 & $\begin{array}{l}\text { Kyoto con comercio de derechos de emisión entre países del Anexo I (ex } \\
\text { Unión Soviética + emisiones) }\end{array}$ & $\checkmark$ & $\checkmark$ & \\
\hline 6 & Kyotr1c & $\begin{array}{l}\text { Kyoto con comercio de derechos de emisión entre países del Anexo I, con } \\
\text { Estados Unidos (ex Unión Soviética = 0) }\end{array}$ & $\checkmark$ & & \\
\hline 7 & Kyotr2a & $\begin{array}{l}\text { Kyoto con comercio de derechos de emisión entre países del Anexo I, sin } \\
\text { Estados Unidos (ex Unión Soviética }=0 \text { ) }\end{array}$ & & & \\
\hline 8 & Kyotr3a & $\begin{array}{l}\text { Kyoto con comercio de derechos de emisión entre países del Anexo I, con } \\
\text { Estados Unidos y G5 (-5\%) }\end{array}$ & $\checkmark$ & & $\checkmark$ \\
\hline 9 & Kyotr3b & $\begin{array}{l}\text { Kyoto con comercio de derechos de emisión entre países del Anexo I, sin } \\
\text { Estados Unidos, pero con G5 }(-5 \%)\end{array}$ & & & $\checkmark$ \\
\hline 10 & Kyotrla1 & $\begin{array}{l}\text { Kyoto con comercio de derechos de emisión entre países del Anexo I, con } \\
\text { Estados Unidos y América Latina }\end{array}$ & $\checkmark$ & & \\
\hline 11 & Kyotrla2 & $\begin{array}{l}\text { Kyoto con comercio de derechos de emisión entre países del Anexo I, sin } \\
\text { Estados Unidos, pero con América Latina }\end{array}$ & & & \\
\hline 12 & Kyowtr1 & $\begin{array}{l}\text { Kyoto con comercio mundial de derechos de emisión (ex Unión Soviética } \\
+ \text { emisiones) }\end{array}$ & $\checkmark$ & $\checkmark$ & \\
\hline 13 & Kyowtr2 & $\begin{array}{l}\text { Kyoto con comercio mundial de derechos de emisión (ex Unión Soviética = } 0 \\
\text { y G5 }=-5 \% \text { ) }\end{array}$ & $\checkmark$ & & $\checkmark$ \\
\hline
\end{tabular}

Fuente: elaboración propia.

Nota: la marca en la columna "Estados Unidos de América" significa que ese país reduce sus emisiones y participa en el comercio de derechos de emisión (en las hipótesis en que este está previsto); la marca en la columna "Ex Unión Soviética" indica que se incluye el "aire caliente" de los países de la ex Unión Soviética; la marca en la columna "Grupo de los 5" indica las hipótesis con una reducción del 5\% en las emisiones del Brasil, China, la India, México y Sudáfrica.

\section{IV \\ Los mercados de carbono y el papel de los países en desarrollo: resultados}

El conjunto de hipótesis analizadas va de la inexistencia de comercio de derechos de emisión a un sistema de comercio mundial, con el objetivo de medir las repercusiones en América Latina y el Caribe. Asimismo, con este estudio se procura determinar el papel que los países en desarrollo (incluidos los países latinoamericanos y caribeños) pueden desempeñar en estas estructuras de negociación. El análisis se concentra en la reducción 
de las emisiones de $\mathrm{CO}_{2}$ (véanse los cuadros 6 y 7) y en la medida del impuesto sobre las emisiones de carbono necesario para lograr esas reducciones (véase el cuadro 8), así como en los efectos en el PIB (véase el cuadro 9) y el bienestar (véanse los cuadros 10 y 11$)^{10}$. Es importante señalar que los valores numéricos de los resultados no son tan relevantes como los signos de los efectos verificados.

\section{Inexistencia de comercio de derechos de emisión: hipótesis de autarquía}

El estudio comienza con los resultados de las diversas hipótesis en las que no se prevé el comercio de derechos de emisión, con la participación de los Estados Unidos de América y sin ella, y con la participación de países en desarrollo seleccionados (Brasil, China, India, México y Sudáfrica) en la reducción de las emisiones. En este caso, los países reducen sus emisiones sin un sistema de comercio de derechos de emisión.

En el cuadro 6 se muestra la variación porcentual de las emisiones de $\mathrm{CO}_{2}$ en todos los países y regiones desde 2001 a 2008-2012. En el caso de los países del Anexo I - a saber: la Unión Europea de 15 miembros, el Japón, el resto de la categoría del Anexo I y los Estados Unidos de América — las primeras dos hipótesis (kyontr1a y kyontr1b) representan el estado actual en que solo los países del Anexo I están obligados a reducir sus emisiones en el marco del Protocolo de Kyoto. La segunda hipótesis es la más cercana a la situación actual, pues los Estados Unidos de América no han ratificado el Protocolo de Kyoto, pero el resto de los países del Anexo I están reduciendo sus emisiones.

En la primera hipótesis, si bien las metas de reducción de las emisiones se alcanzan en los países del Anexo I, estas aumentan en todos los demás países, en algunos casos casi un $3 \%$. Este efecto, denominado fuga de carbono, es uno de los problemas de un sistema que carece de compromisos a nivel mundial, pues mientras algunos países pueden reducir sus emisiones, otros, sin ningún tipo de restricciones vinculantes, pueden aumentarlas. En la segunda hipótesis, en la que no se reducen las emisiones de los Estados Unidos de América, la variación en las emisiones de los países que no figuran en el Anexo I es positiva, pero inferior

\footnotetext{
10 Los cambios en el bienestar solo tienen en cuenta los efectos derivados de las hipótesis simuladas en este trabajo. En los resultados no se incluyen los efectos en el bienestar que derivan de los daños causados por el cambio climático, medidas de adaptación u otras políticas de mitigación.
}

a la de la primera hipótesis (y de hecho es negativa en el caso de la India).

Cuando en algunos países en desarrollo seleccionados (G5) se reducen voluntariamente las emisiones un 5\% (kyontr2a y kyontr2b), aumentan las emisiones de los países que no figuran en el Anexo I. Ese incremento es mayor que en las dos primeras hipótesis, pues a medida que los países del G5 disminuyen sus emisiones se amplía el margen para aumentos en los países no incluidos en el Anexo ${ }^{11}$.

El costo relacionado con estas reducciones se detalla en el cuadro 8. En la primera hipótesis, el equivalente del impuesto sobre las emisiones de carbono (en dólares por tonelada) oscila entre 9,72 dólares en la Unión Europea de 15 miembros y 36,2 dólares en el Japón. En los Estados Unidos de América y en el resto del Anexo I, el equivalente del impuesto sobre las emisiones de carbono es de casi 22 dólares por tonelada. Cabe señalar que el costo de la reducción de un 5\% de las emisiones en los países del G5 es más bajo que para cualquiera de los del Anexo I. El costo más bajo se registra en la India (menos de 1 dólar por tonelada), seguida por China (1,5 dólares-1,6 dólares por tonelada) y Sudáfrica (4 dólares por tonelada). El costo en los dos países latinoamericanos, el Brasil y México, es más alto y similar al de la Unión Europea, entre 7 dólares y 9 dólares por tonelada. Estos resultados reflejan la ventaja de los países en desarrollo con respecto a los países desarrollados en cuanto a la reducción de las emisiones de $\mathrm{CO}_{2}$ a un costo inferior. Este aspecto se analiza más adelante con mayor detalle.

Los efectos en el PIB y el bienestar se muestran en los cuadros 9 y 10, respectivamente. En el caso del PIB, el signo de las variaciones es en realidad más significativo que su magnitud ${ }^{12}$. Como se esperaba, la reducción de las emisiones tiene un efecto negativo marginal en el PIB de los países del Anexo I en todas las hipótesis, que incluso desaparece cuando se considera a los Estados Unidos de América fuera del Protocolo de Kyoto. También cabe destacar que cuando se reducen las emisiones de los Estados Unidos de América mediante recortes en el consumo de productos de energía, se observan efectos negativos directos en los países exportadores de energía, en particular la República Bolivariana de Venezuela. La disminución de las emisiones en el Brasil, China, la India, México y Sudáfrica tiene un efecto negativo marginal en

\footnotetext{
11 Al no haber comercio, cada país o región constituye su propio bloque y los resultados del cuadro 6 son iguales a los del cuadro 7 .

${ }^{12}$ Las variaciones en el PIB son bastante pequeñas, sobre todo debido a la dimensión de los shocks y a la naturaleza estática del modelo en sí, que no capta la dinámica de la reducción de las emisiones de carbono.
} 


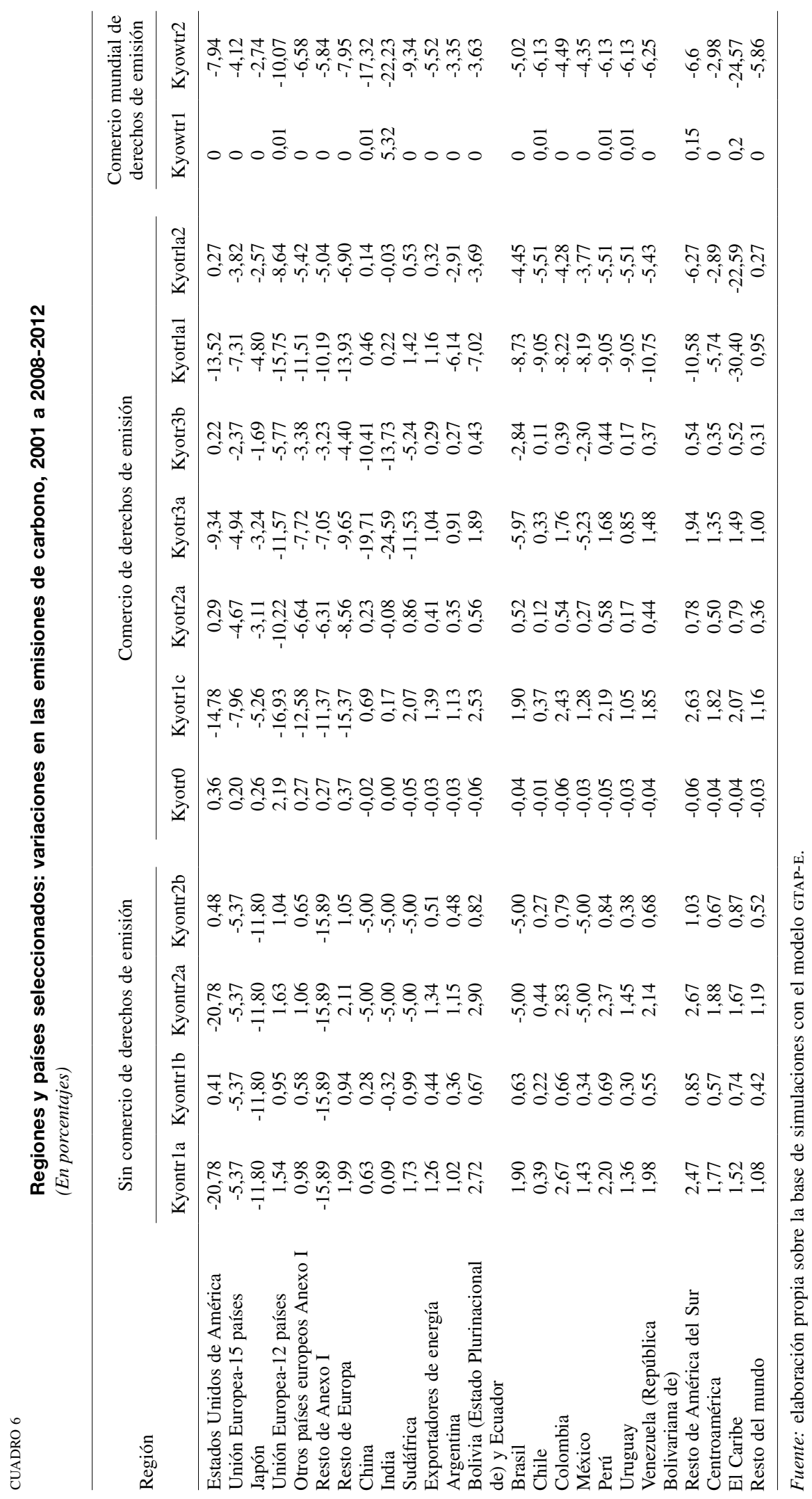




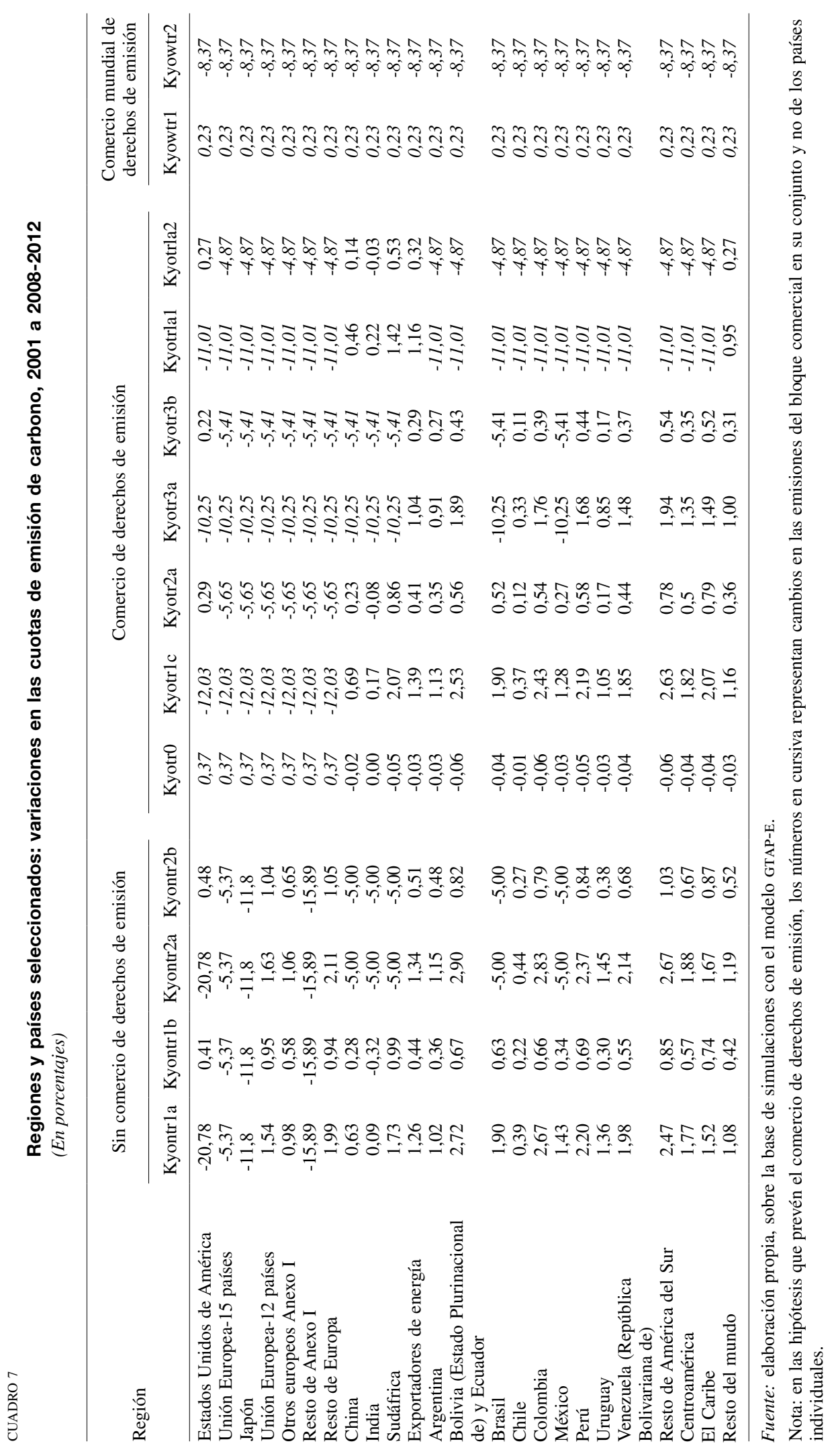




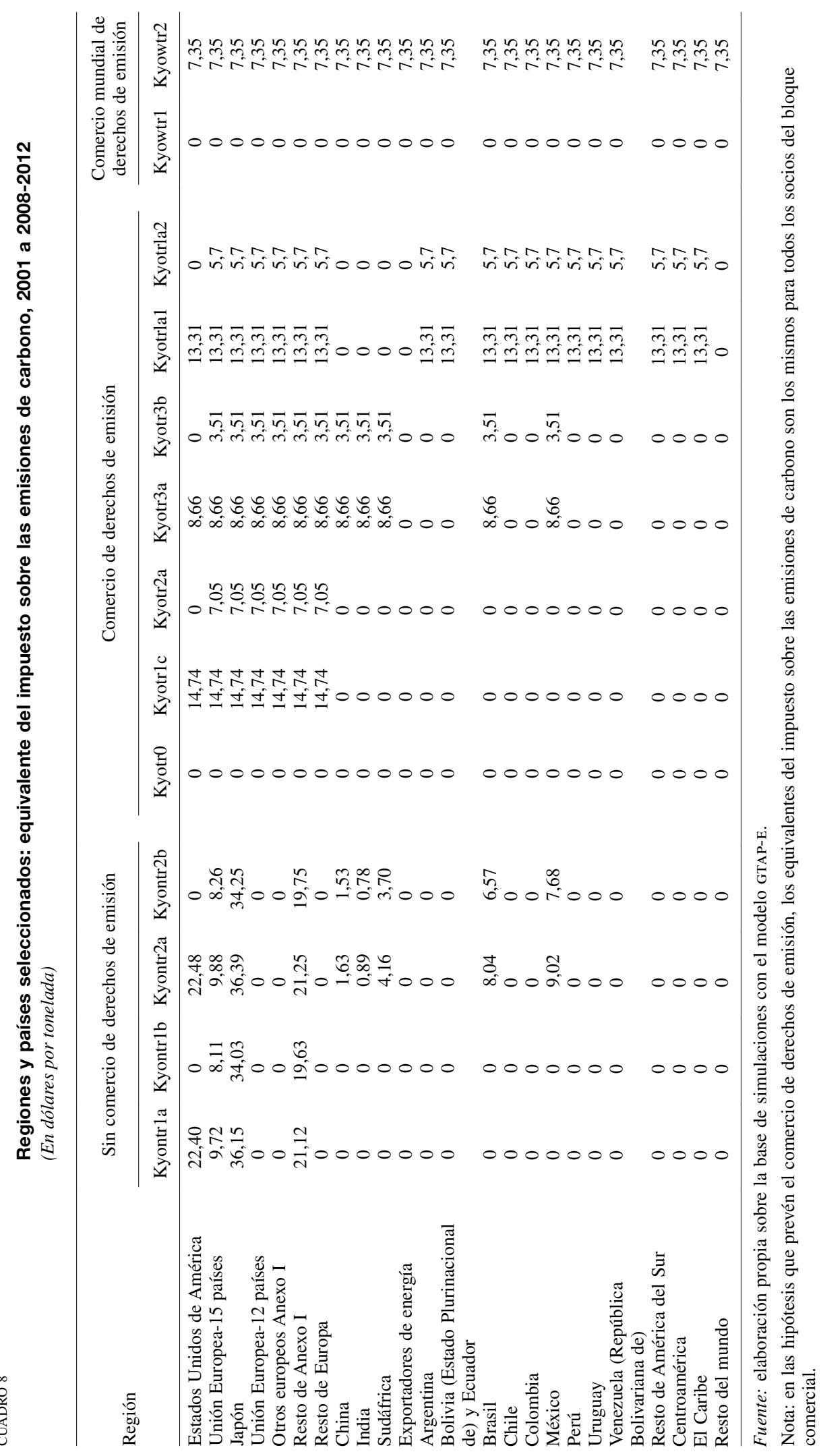




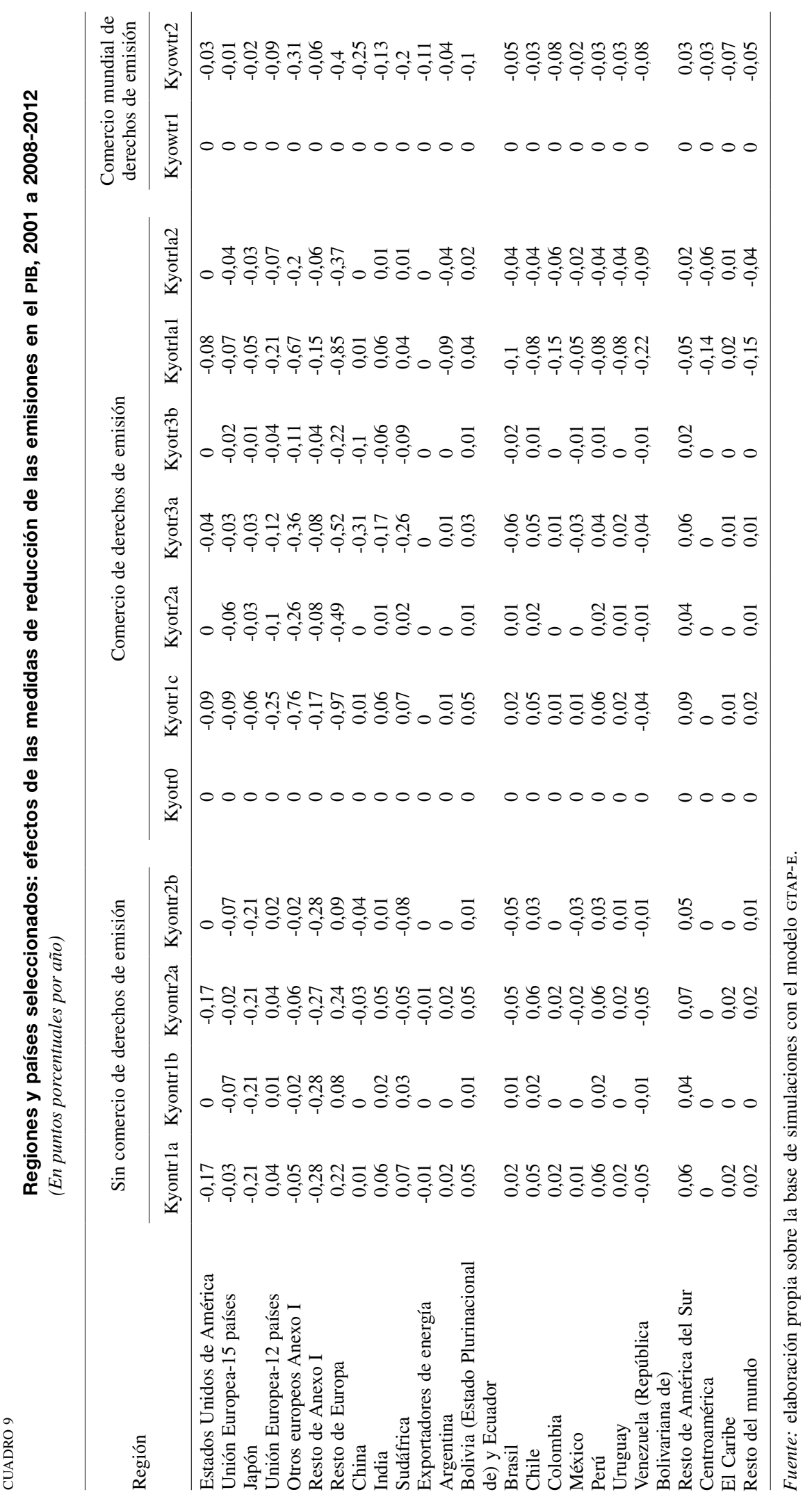




\begin{tabular}{|c|c|c|c|c|c|}
\hline 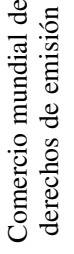 & 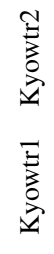 & 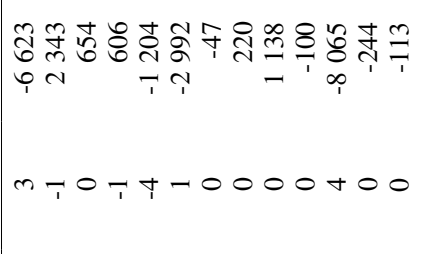 & 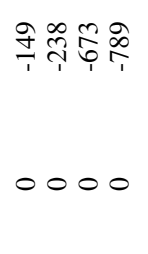 & 00 & 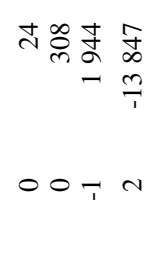 \\
\hline & 吾 & 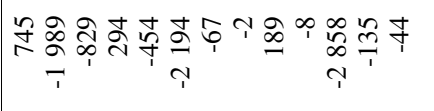 & 익 & স্ন & 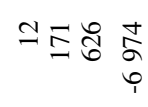 \\
\hline & $\frac{\bar{\sigma}}{\vec{E}}$ & 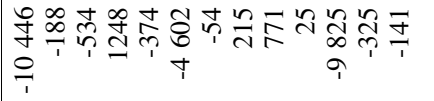 & 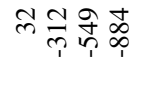 & $\infty \stackrel{n}{n}$ & 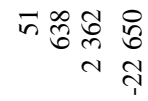 \\
\hline 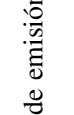 & 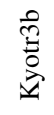 & 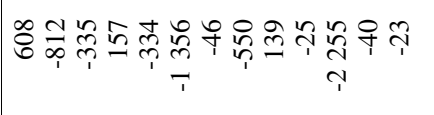 & م્o & $\vec{n}$ in & 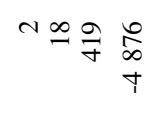 \\
\hline 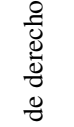 & 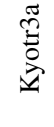 & 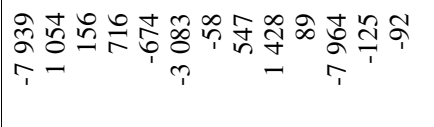 & क् & $\stackrel{\infty}{i \underset{\infty}{ \pm}}$ & 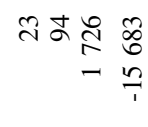 \\
\hline 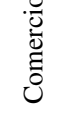 & 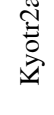 & 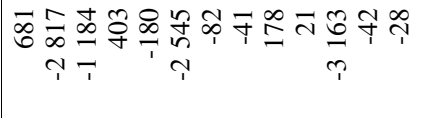 & ¿ స్ర & $\infty$ & 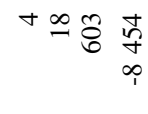 \\
\hline & 竞 & 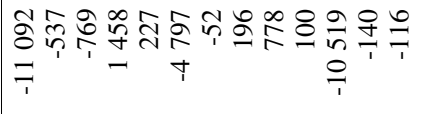 & 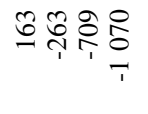 & $\infty$ ఎ స్ন & 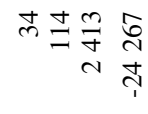 \\
\hline & 吾 & 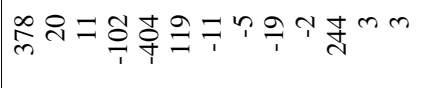 & 욕 & Nin & † \\
\hline :ర్ & 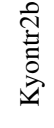 & 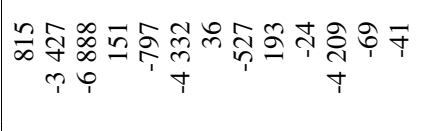 & 욱위 & $\vec{\sigma} \delta$ & 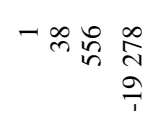 \\
\hline 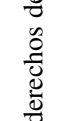 & 壱 & 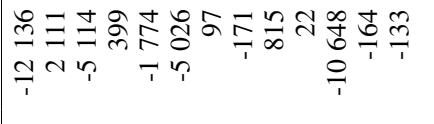 & 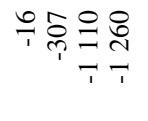 & $\vec{্}$ & 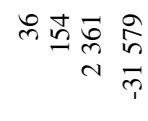 \\
\hline 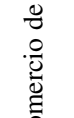 & $\overline{\vec{E}}$ & 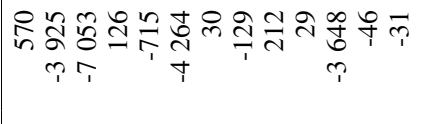 & 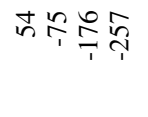 & ले & 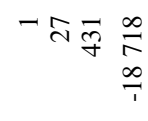 \\
\hline$=$ & $\overrightarrow{\underline{\Xi}}$ & 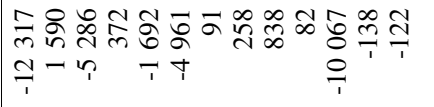 & 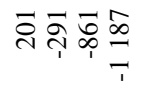 & nి ః్ & 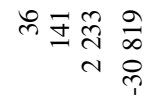 \\
\hline & & 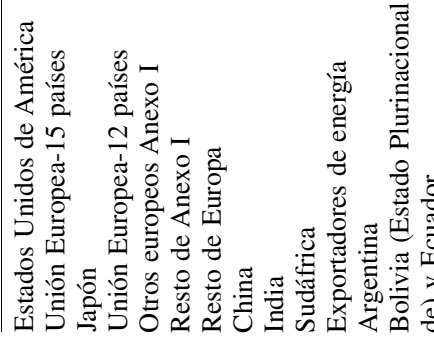 & 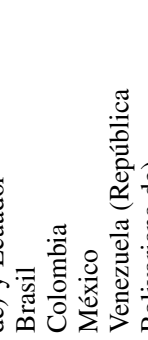 & 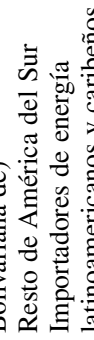 & 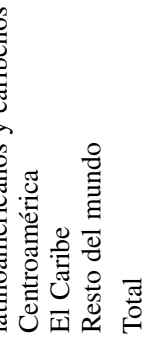 \\
\hline
\end{tabular}




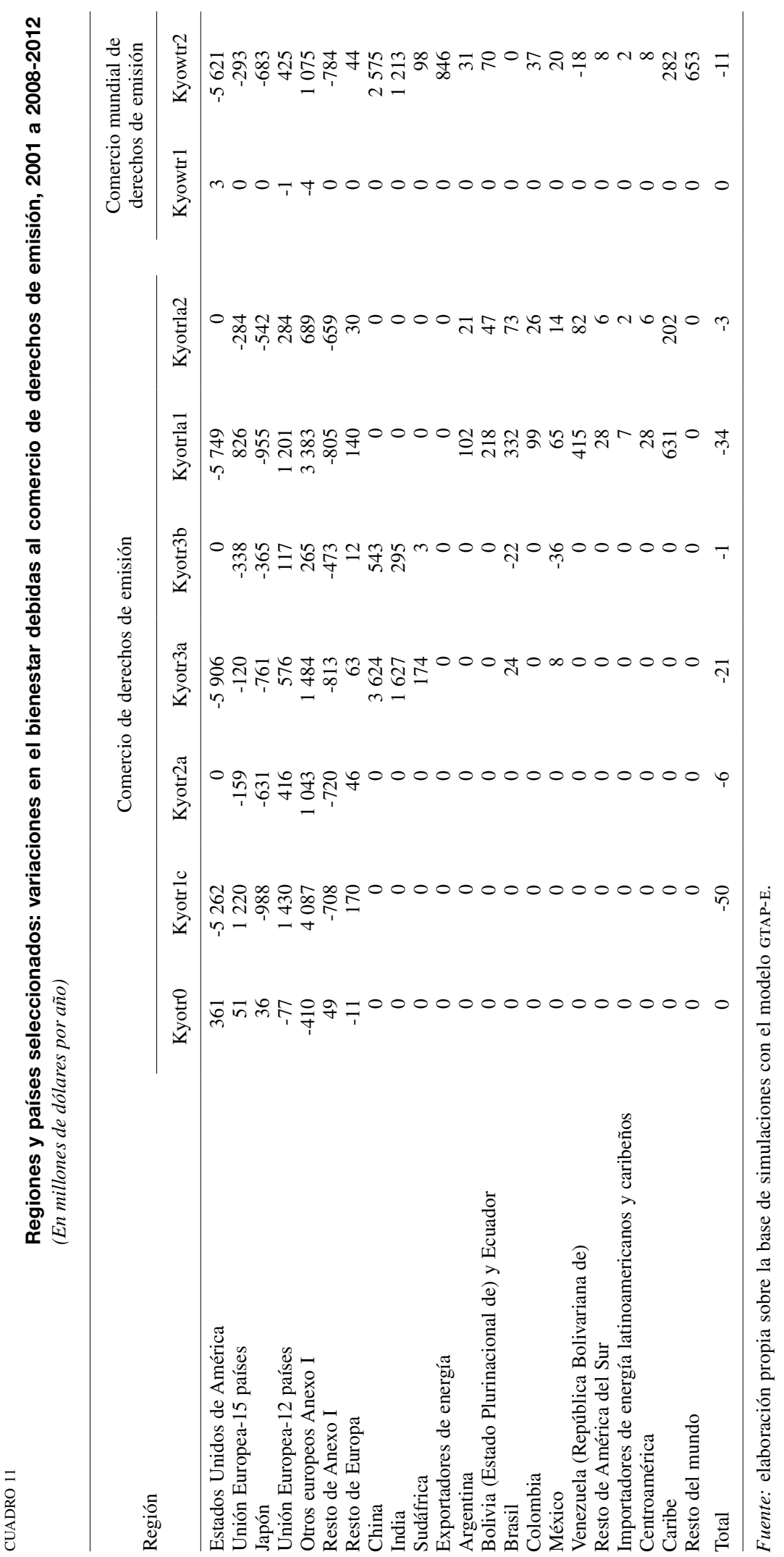


el PIB de todos ellos, con excepción de la India. Como ya se mencionó, el costo de la reducción de las emisiones en ese país es el más bajo con relación a todos los países desarrollados y en desarrollo examinados, de modo que el impacto en el PIB es mínimo.

Con respecto a las variaciones en el bienestar, en todas las hipótesis en que no hay comercio de derechos de emisión se prevén pérdidas de entre 19.000 millones de dólares y 20.000 millones de dólares por año. Las pérdidas menores se registran cuando no se incluye a los Estados Unidos de América. En la primera hipótesis, un tercio de las pérdidas de bienestar corresponde a los países en desarrollo. La mayoría de los países afectados son exportadores de energía (con una pérdida de 10.000 millones de dólares), donde el impacto es peor que en el Japón o en el resto del Anexo I, y la mayor parte de las pérdidas de bienestar derivan de los términos de intercambio. Por ejemplo, en la República Bolivariana de Venezuela, exportador de energía y el país latinoamericano con la mayor pérdida de bienestar, este resultado obedece sobre todo a las variaciones en los términos de intercambio en los sectores del crudo y los derivados del petróleo. En la segunda hipótesis, en que no se reducen las emisiones de los Estados Unidos de América, se observa un efecto directo en la mayoría de los países en desarrollo. Si bien la pérdida de bienestar potencial para los países exportadores de energía disminuye, esto se compensa por el efecto en los países importadores de energía como el Brasil, China y la India, donde se reducen las ganancias de bienestar. Este efecto en los países importadores de energía deriva de los términos de intercambio, pues los precios de los productos de energía como el crudo o los derivados del petróleo no disminuyen. Sin embargo, las variaciones en el bienestar relacionadas con el comercio de derechos de emisión son positivas para la mayoría de los países en desarrollo, incluso en el caso en que el G5 mitigue sus emisiones, a menos que los Estados Unidos de América no participen en el mercado. En ese caso, tanto el Brasil como México tienen menos ventajas comparativas que China y la India, y pueden sufrir algunas pérdidas de bienestar.

Por último, cuando aminoran las emisiones de los países del G5 se observa un efecto negativo en el bienestar del Brasil, China y México. Las pérdidas de bienestar varían con las oportunidades comerciales y la participación de los Estados Unidos de América. Cuando se reducen las emisiones de $\mathrm{CO}_{2}$ de ese país, China, la India y Sudáfrica se benefician, porque sus oportunidades de mitigación les proporcionan ventajas comparativas con respecto a los Estados Unidos de América, que producen efectos positivos en el bienestar. Por otra parte, el Brasil no resulta afectado, mientras que México sufre pérdidas de bienestar mayores. Los estrechos lazos entre las economías mexicana y estadounidense y el papel de México como importante exportador de energía hacen que los compromisos de reducción de las emisiones de los Estados Unidos de América también repercutan en el bienestar mexicano a través del canal de los términos de intercambio, sumándose a los efectos de los propios compromisos de México.

\section{Comercio de derechos de emisión: países del Anexo I y países en desarrollo}

En esta subsección se analiza el comercio de derechos de emisión entre los países del Anexo I y se incluye la participación de países en desarrollo seleccionados en la estructura de negociación, en particular el G5 y los países latinoamericanos. Cuando los países del Anexo I reducen sus emisiones y se incluye el "aire caliente" de los países de la ex Unión Soviética (kyotr0), la variación en las emisiones de $\mathrm{CO}_{2}$ en todos los países equivale casi a cero (véase el cuadro 6). La variación en las emisiones a nivel del bloque de los países del Anexo I es del 0,37\% (véase el cuadro 7). En otras palabras, la variación total en las emisiones —al incluir las reducciones de los Estados Unidos de América, el Japón, la Unión Europea de 15 miembros y otros países del Anexo I y el "aire caliente" de los países de la ex Unión Soviética- es casi cero en una hipótesis de comercio de derechos de emisión dentro de ese conjunto de países. Como consecuencia, el costo efectivo de la disminución de las emisiones es casi igual a cero (véase el cuadro 8). Debido a que las variaciones en las emisiones son cercanas a cero, lo mismo ocurre con las variaciones en el PIB. Con respecto al bienestar, se registra un efecto positivo a nivel mundial de 208 millones de dólares por año. En cuanto a las variaciones en el bienestar derivadas del comercio de derechos de emisión (véase el cuadro 11), el efecto neto es cero, y las ganancias de bienestar para los países del Anexo I distintos de los de la ex Unión Soviética se compensan con las pérdidas de bienestar de estos últimos. Estas ganancias de bienestar y la neutralidad del comercio de derechos de emisión demuestran las ventajas de implementar un sistema de comercio con respecto a no implementarlo.

En la segunda y la tercera hipótesis se examina el caso del comercio de derechos de emisión entre los países del Anexo I (con y sin los Estados Unidos de América), pero sin incluir el "aire caliente" de los países de la ex Unión Soviética. Estas dos hipótesis permiten poner a prueba el caso en que los países de la ex Unión 
Soviética mantienen igual su cuota de emisiones. Los resultados muestran que la variación en las emisiones de $\mathrm{CO}_{2}$ difieren entre las dos hipótesis (véase el cuadro 6). Cuando los Estados Unidos de América disminuyen sus emisiones también participan en el mercado de carbono. La reducción de las emisiones de los países del Anexo I es mayor cuando los Estados Unidos de América participan que cuando no lo hacen. Asimismo, cuanto más se aminoran las emisiones de los países del Anexo I, mayor es la fuga de carbono en los países en desarrollo.

La reducción a nivel de bloque es mayor con la participación de los Estados Unidos de América en el mercado de carbono (12\%) que sin ella $(5,7 \%)$. Este nivel de reducción se relaciona directamente con el nivel del impuesto sobre las emisiones de carbono necesario para disminuir las emisiones de $\mathrm{CO}_{2}$. La reducción de las emisiones de $\mathrm{CO}_{2}$ es mayor cuando los Estados Unidos de América participan en el mercado de carbono, con un equivalente de impuesto sobre las emisiones de 14,74 dólares por tonelada. Al contrario, cuando los Estados Unidos de América no participan en el mercado de carbono, tanto la disminución de las emisiones de $\mathrm{CO}_{2}$ como el nivel del impuesto necesario para reducirlas son inferiores ( 7,05 dólares por tonelada).

Es importante señalar que estos equivalentes del impuesto sobre las emisiones de carbono son inferiores a los impuestos resultantes cuando no hay un sistema de comercio de derechos de emisión de $\mathrm{CO}_{2}$, por lo que se destaca la relevancia de implementar un mercado de carbono. Con respecto al bienestar, al igual que en el caso anterior, la reducción de las emisiones en los Estados Unidos de América se traduce en una pérdida de bienestar que también afecta directamente a los países exportadores de energía. Sin embargo, las pérdidas de bienestar son menores que cuando no hay un sistema de comercio. En cuanto a las variaciones en el bienestar derivadas del comercio de derechos de emisión, los resultados muestran que las ganancias de bienestar para los otros países del Anexo I disminuyen cuando los Estados Unidos de América no participan, pues su ausencia aminora el tamaño del mercado.

En las cuatro hipótesis siguientes se incorpora la participación de países en desarrollo seleccionados en el mercado de carbono. En las primeras dos se incluye al Brasil, China, la India, México y Sudáfrica (G5), mientras que en las dos últimas se incorpora a los países de América Latina y el Caribe. Los resultados señalan que la participación de los países en desarrollo disminuye el costo del equivalente del impuesto. El equivalente del impuesto sobre las emisiones de carbono se reduce casi a la mitad cuando se incluye a los países del G5 y alrededor de 1 dólar por tonelada cuando participan los países latinoamericanos. Esto puede ser un indicio del peso de estos últimos con respecto a otros países en desarrollo. El efecto en el bienestar es el mismo, con mayores variaciones positivas cuando participan los países en desarrollo. El comercio de derechos de emisión es una fuente importante de variaciones positivas en el bienestar. En China y la India se registran variaciones positivas en términos generales, debido a que captan una gran proporción del mercado gracias al bajo costo de la reducción de emisiones en ambos países. $\mathrm{Al}$ igual que antes, las ganancias en el bienestar disminuyen cuando los Estados Unidos de América no reducen sus emisiones y no participan en el comercio de derechos de emisión, pues el mercado de carbono es más pequeño. Cuando se presume un mercado mundial de derechos de emisión, los costos son menores y el volumen de mercado es más pequeño que cuando se presume el comercio solo entre países con metas de emisión cuantificadas (países del Anexo I). Al mismo tiempo, cuando en el análisis se incluyen todos los gases de efecto invernadero, los costos y los precios de los permisos disminuyen con respecto a los modelos que solo tienen en cuenta las emisiones de $\mathrm{CO}_{2}$. Así, las limitaciones a la participación aumentarían los costos de reducción.

Por otra parte, la participación de numerosos países en desarrollo aminora los costos de cumplimiento de los países del Anexo I y aumenta los beneficios para los países de la Organización para la Cooperación y el Desarrollo Económicos (OCDE). Los países en desarrollo también se benefician, pues obtienen recursos financieros adicionales y reducen su nivel de emisiones de carbono de referencia. Sin embargo, las ganancias para los países de la ex Unión Soviética disminuyen a medida que se incrementa la participación de los países en desarrollo, lo que puede tener importantes consecuencias en materia de normas y reglas que rigen la admisión de nuevos países a los sistemas de comercio de derechos de emisión.

\section{Comercio mundial de derechos de emisión}

Con el comercio mundial de derechos de emisión, la variación en las emisiones en la primera hipótesis (con reducciones en los países del Anexo I y "aire caliente" de los países de la ex Unión Soviética) es de casi cero, mientras que a nivel de bloque las cuotas de emisiones aumentan solo un $0,23 \%$, con un equivalente del impuesto sobre las emisiones de carbono igual a cero. En virtud de estos pequeños cambios en las emisiones, casi no hay variaciones en el PIB o el bienestar. Al comparar esta hipótesis con las otras dos que incorporan "aire 
caliente" (kyontr1a y kyotr0) se observa que, a diferencia de las pérdidas de bienestar en la hipótesis de autarquía, el comercio de derechos de emisión mitiga los efectos económicos negativos que la reducción de las emisiones puede tener en los países desarrollados y en desarrollo. Los países del Anexo I pueden diminuir sus emisiones sin obstaculizar el crecimiento económico o el bienestar, lo que refleja la eficacia de un sistema de comercio global.

En la hipótesis en que se reducen las emisiones en los países en desarrollo (G5) y se elimina el "aire caliente", la exclusión de los créditos de emisión de los países de la ex Unión Soviética de las cuentas significa que otros países alrededor del mundo deben aminorar sus emisiones. Esto muestra la importancia del supuesto del "aire caliente" cuando se modelan mercados de carbono, pues permite a los diferentes países, y en particular a los del Anexo I que no formaban parte de la Unión Soviética, cumplir con sus compromisos de reducción mediante negociaciones con los países de la ex Unión Soviética. Al eliminar este mecanismo, los países en todo el mundo deben disminuir sus emisiones colectivas casi un 9\% (véase el cuadro 7).

En consecuencia, tanto los países desarrollados como aquellos en desarrollo reducen sus emisiones entre un $3 \%$ y un $25 \%$. Entre los países en desarrollo, algunas de las mayores reducciones se registran en importantes actores como China (17\%), la India (22\%) y Sudáfrica (9\%). En todos los países latinoamericanos (pero no en los del Caribe), las emisiones descienden entre un $3 \%$ y un $6 \%$.

En lo que respecta al bienestar, la reducción de las emisiones provoca pérdidas en los países del Anexo I y en los exportadores de energía. Los países en desarrollo, como China y la India, presentan ganancias de bienestar, al igual que los países del Anexo I como el Japón y la Unión Europea de 15 miembros. Sin embargo, es relevante señalar que el comercio de derechos de emisión se convierte en una importante fuente de ganancias de bienestar para China y la India (véase el cuadro 11), que equivalen a 2.600 millones de dólares y 1.200 millones de dólares, respectivamente. Como ya se mencionó, es más económico disminuir las emisiones en estos dos países que en otros países en desarrollo, lo que podría explicar por qué estos captan la mayor parte de las ganancias de bienestar derivadas del comercio de derechos de emisión. En el caso de los países latinoamericanos como el Brasil y México, las ganancias de bienestar derivadas del mercado de carbono son pequeñas y no compensan las posibles pérdidas de otras fuentes, como los términos de intercambio o la asignación de recursos.

\section{V}

\section{Conclusiones y repercusiones en las políticas}

Visto que el cambio climático se debe a las emisiones antropógenas, la humanidad necesita encontrar soluciones para impedir el continuo aumento de la temperatura media global, la modificación de los patrones de precipitación y la elevación del nivel del mar, entre otros fenómenos que dañarían de modo irreversible la resiliencia del planeta. De acuerdo con las previsiones más probables, la temperatura media aumentará entre $1{ }^{\circ} \mathrm{C}$ y $4{ }^{\circ} \mathrm{C}$ en este siglo. La mitigación de las emisiones de gases de efecto invernadero para estabilizar el clima es fundamental y requiere un acuerdo a nivel mundial. Las medidas necesarias representan un desafío para el modelo económico actual y tendrán que superar con creces los compromisos aceptados en el marco del Protocolo de Kyoto. Si bien el establecimiento de nuevos compromisos para los países desarrollados y de aportes a la mitigación a nivel mundial será imprescindible, estos pueden traer aparejados costos a corto plazo. La mitigación requerirá diversos instrumentos de eficiencia y flexibilidad variables y la distribución de ganadores y perdedores en todo el mundo será desigual. No obstante, el impacto del cambio climático en ausencia de medidas sería aún mucho más costoso, y los países afectados deberían ser compensados económicamente por sus repercusiones.

En este trabajo se simularon y analizaron diferentes escenarios de reducción de emisiones de carbono y estructuras para el comercio de los derechos de emisión (con sus respectivos equivalentes de impuestos sobre las emisiones de $\mathrm{CO}_{2}$ ), y sus repercusiones en las economías y el bienestar de los países desarrollados y en desarrollo, en particular los de América Latina y el Caribe. Los resultados presentan varios hechos estilizados coherentes con estudios anteriores. En primer lugar, la participación de los Estados Unidos de América es crucial para disminuir las emisiones en todo el mundo, 
así como para determinar el costo de la reducción de las emisiones. Es imprescindible entonces que los mercados de carbono incluyan a los Estados Unidos de América, que es el segundo mayor emisor después de China, al tiempo que la mayoría de los países en desarrollo también se beneficiarían de mejoras en la competitividad y de la participación en ese sistema de comercio de reducciones.

En segundo lugar, el efecto del "aire caliente" de los países de la ex Unión Soviética constituye un factor importante en el mercado de comercio de derechos de emisión. Suponiendo las tasas de reducción de las emisiones de $\mathrm{CO}_{2}$ simuladas en este trabajo, su inclusión disminuiría el costo de la mitigación a corto plazo. Sin embargo, considerando que el superávit de emisiones mencionado es insuficiente en el largo plazo, el efecto del "aire caliente" no ofrecerá el mismo nivel de flexibilidad en los regímenes de comercio de emisiones, dado que los países beneficiados tendrán que realizar esfuerzos de mitigación sin emisiones sobrantes.

En tercer lugar, la participación de los países en desarrollo es fundamental para aminorar los costos de reducción de las emisiones de $\mathrm{CO}_{2}$. Este efecto se amplía cuando algunos de esos países en desarrollo también asumen compromisos de mitigación (se simularon iniciativas de mitigación del Brasil, China, la India, México y Sudáfrica), disminuyendo aún más de ese modo los costos de mitigación.

El impacto económico en los países en desarrollo, que es siempre muy pequeño, varía según se trate de países exportadores o importadores de energía. Los resultados también están influenciados por la participación de los Estados Unidos de América en los esfuerzos para aminorar las emisiones. En los países exportadores de energía se observan pérdidas de bienestar causadas sobre todo por un deterioro en los términos de intercambio, debido a que los países del Anexo I reducen sus emisiones disminuyendo el consumo de productos del sector de energía, como carbón, gas, crudo y derivados del petróleo. Esto afecta los términos de intercambio de los países exportadores de energía, pues los precios de sus productos bajan con respecto a los precios de importación. El impacto mayor de los términos de intercambio se registra en los países latinoamericanos exportadores de energía como la Argentina, Colombia, México y la República Bolivariana de Venezuela, en virtud de su estrecha relación con los Estados Unidos de América como socio comercial. Sin embargo, las variaciones en el bienestar debidas a la participación en un sistema de comercio de derechos de emisión son en general positivas para los países latinoamericanos (a menos que los Estados Unidos de América no participen), incluso cuando se han comprometido a rebajar sus propias emisiones. La República Bolivariana de Venezuela es el único país que podría sufrir a causa de un sistema mundial de comercio de emisiones con compromisos para todos los principales contaminadores (países del Anexo I del Protocolo de Kyoto y del G5).

Los resultados ponen de relieve el importante papel que los países en desarrollo pueden desempeñar en el mercado de derechos de emisión de carbono y en la determinación del costo de la reducción de las emisiones. Sin embargo, el estudio también revela que para algunos países en desarrollo exportadores de energía el impacto de la disminución de las emisiones de carbono puede ser negativo, manteniéndose constantes las demás condiciones, pues la demanda de sus productos podría disminuir. No obstante, es importante señalar que en este trabajo no se tuvo en cuenta el mecanismo para un desarrollo limpio, que podría aminorar algunos de esos efectos negativos para los países en desarrollo. Por último, cabe destacar que en esta evaluación no se incluyeron los efectos dinámicos. De este modo, los incentivos a largo plazo que un impuesto sobre las emisiones de carbono podría crear para la asignación de inversiones en todo el mundo y entre sectores económicos, así como las variaciones futuras en la competitividad deben ser objeto de futuras investigaciones. Los ganadores y perdedores de una estructura de comercio pueden cambiar dependiendo de la capacidad de los países para adaptar sus economías en un contexto dinámico. Sin embargo, los costos a corto plazo son lo suficientemente bajos para justificar la toma de medidas, como también constituiría una ventaja el establecimiento de estructuras de negociación que proporcionen flexibilidad.

Con respecto a las repercusiones normativas que pueden deducirse de este análisis, se recomienda a las autoridades de los países en desarrollo tener en cuenta los siguientes tres elementos: i) los efectos potencialmente negativos que la reducción de las emisiones por las naciones industrializadas podría producir en sus economías a corto plazo y los mecanismos que se pueden utilizar para mitigar algunos de esos efectos; ii) el papel que pueden desempeñar en los mercados de carbono internacionales cuando participan en las negociaciones de las Conferencias de las Partes (CP) de la CMNUCC cada año, y iii) el posible papel y los beneficios para los países en desarrollo de otros mecanismos de flexibilidad previstos en el Protocolo de Kyoto. 


\section{Bibliografía}

AIE (Agencia Internacional de la Energía) (2010a), CO2 Emissions from Fuel Combustion 2010-Highlights, París, Organización para la Cooperación y el Desarrollo Económicos (OCDE)/ Agencia Internacional de la Energía (AIE).

(2010b), Reviewing Existing and Proposed Emissions Trading Systems, París, Organización para la Cooperación y el Desarrollo Económicos (OCDE)/Agencia Internacional de la Energía (AIE).

Anger, N. (2008), "Emissions trading beyond Europe: Linking schemes in a post-Kyoto world", Energy Economics, vol. 30, $\mathrm{N}^{\circ} 4$, Amsterdam, Elsevier.

Beckman, J.F. y T.W. Hertel (2009), "Why previous estimates of the cost of climate mitigation are likely too low", GTAP Working Papers, $\mathrm{N}^{\circ}$ 54, West Lafayette, Universidad de Purdue.

Birur, D., T.W. Hertel y W. Tyner (2007), "Impact of biofuel production on world agricultural markets: A computable general equilibrium analysis", GTAP Working Papers, $\mathrm{N}^{\circ} 53$, West Lafayette, Universidad de Purdue.

Burniaux, J.M. y T.P. Truong (2002), "GTAP-E: An energyenvironmental version of the GTAP model", GTAP Technical Paper, $\mathrm{N}^{\circ} 16$, West Lafayette, Universidad de Purdue.

Capoor, K. y P. Ambrosi (2008), State and Trends of the Carbon Market 2008, Washington, D.C., Banco Mundial, mayo.

CEPAL (Comisión Económica para América Latina y el Caribe) (2009a), Economics of Climate Change in Latin America and the Caribbean. Summary 2009 (LC/G.2425), Santiago de Chile, noviembre.

(2009b), "La economía del cambio climático en Chile. Síntesis" (LC/W.288), Santiago de Chile.

CMNUCC (Convención Marco de las Naciones Unidas sobre el Cambio Climático) (2007), "National greenhouse gas inventory data for the period 1990-2005" (FCCC/SBI/2007/30) [en línea] http:// unfecc.int/resource/docs/2007/sbi/eng/30.pdf.

(1997), "Protocolo de Kyoto de la Convención Marco de las Naciones Unidas sobre el Cambio Climático" [en línea] http://unfccc.int/resource/docs/convkp/kpspan.pdf.

Dagoumas, A.S., G.K. Papagiannis y P.S. Dokopoulos (2006), "An economic assessment of the Kyoto Protocol application", Energy Policy, vol. 34, $\mathrm{N}^{\circ} 1$, Amsterdam, Elsevier.

De la Torre, A., P. Fajnzylber y J. Nash (2009), Low Carbon, High Growth: Latin American Responses to Climate Change-An Overview, Washington, D.C., Banco Mundial.

Evans, M. (2003), "Emissions trading in transition economies: The link between international and domestic policy", Energy Policy, vol. 31, $\mathrm{N}^{\circ}$ 9, Amsterdam, Elsevier.

Flachsland, C., R. Marschinski y O. Edenhofer (2009), "Global trading versus linking: Architectures for international emissions trading”, Energy Policy, vol. 37, 우 5, Amsterdam, Elsevier.

Hamasaki, H. (2004), "Japanese strategy on climate change to achieve the Kyoto Target with steady economic development-An investigation by using the dynamic version of GTAP-E model", documento presentado en la Séptima Conferencia Anual sobre Análisis Económico Global, Washington, D.C.

Hamasaki, H. y T. Truong (2001), "The costs of green house gas emission reductions in the Japanese economy-An investigation using the GTAP-E model", documento presentado en la Cuarta Conferencia Anual sobre Análisis Económico Global, Universidad de Purdue.

Hertel, T.W. (ed.) (1997), Global Trade Analysis: Modeling and Applications, Nueva York, Cambridge University Press.

Hertel, T.W. y otros (2006), "The role of global land use in determining greenhouse gases mitigation costs", GTAP Working Papers, $\mathrm{N}^{\circ} 2230$, Universidad de Purdue.

Houba, H. y H. Kremers (2007), "Bargaining for an efficient and fair allocation of emissions permits to developing countries", documento presentado en la GTAP Conference, 7 a 9 de junio, Universidad de Purdue.

IPCC (Grupo Intergubernamental de Expertos sobre el Cambio Climático) (2007), Climate Change 2007: The Physical Science Basis. Contribution of Working Group I to the Fourth Assessment Report of the IPCC, Cambridge, Cambridge University Press.

Johnson, T. y otros (2009), Low-Carbon Development for Mexico, Washington, D.C., Banco Mundial.

Lee, H. (2008), "An emissions data base for integrated assessment of climate change policy using GTAP", GTAP Resources, $\mathrm{N}^{\circ} 1143$, West Lafayette, Universidad de Purdue.

Ludeña, C.E. (2007), " $\mathrm{CO}_{2}$ Emissions by fuel and user for GTAP-E", GTAP Resource, $\mathrm{N}^{\circ}$ 2508, West Lafayette, Universidad de Purdue.

McDougall, R. y A. Golub (2009), "GTAP-E release 6: A revised energy-environmental version of the GTAP model", GTAP Research Memorandum, $\mathrm{N}^{\circ}$ 15, West Lafayette, Universidad de Purdue.

Medvedev, D. y D. van der Mensbrugghe (2010), "Climate change in Latin America: Impacts and mitigation policy options", Modeling Public Policies in Latin America and the Caribbean, Carlos de Miguel y otros (ed.), Santiago de Chile, Comisión Económica para América Latina y el Caribe (CEPAL)/Banco Interamericano de Desarrollo (BID).

Nijkamp, P., S. Wang y H. Kremers (2005), "Modeling the impacts of international climate change policies in a CGE context: The use of the GTAP-E model”, Economic Modelling, vol. 22, $\mathrm{N}^{\circ} 6$, Amsterdam, Elsevier.

OCDE (Organización para la Cooperación y el Desarrollo Económicos) (2013), "Climate and carbon: Aligning prices and policies", OECD Environment Policy Paper, $\mathrm{N}^{\circ} 01$, octubre.

O'Ryan, R., C. de Miguel y S. Miller (2000), "Ensayo sobre equilibrio general computable: Teoría y aplicaciones", Documentos de Trabajo, $\mathrm{N}^{\circ} 73$, Santiago de Chile, Centro de Economía Aplicada.

Samaniego, J. (coord.) (2009), "Cambio climático y desarrollo en América Latina y el Caribe: Una reseña" (LC/W.232), Santiago de Chile, Comisión Económica para América Latina y el Caribe (CEPAL), febrero.

Schuschny, A., J. Durán y C. de Miguel (2007), "El modelo GTAP y las preferencias arancelarias en América Latina y el Caribe: Reconciliando su año base con la evolución reciente de la agenda de liberalización regional", serie Manuales, $\mathrm{N}^{\circ} 53$ (LC/L.2679-P), Santiago de Chile, Comisión Económica para América Latina y el Caribe (CEPAL). Publicación de las Naciones Unidas, $\mathrm{N}^{\circ}$ de venta: S.07.II.G.29.

Springer, U. (2003), "The market for tradable GHG permits under the Kyoto Protocol: a survey of model studies", Energy Economics, vol. 25, $\mathrm{N}^{\circ}$ 5, Amsterdam, Elsevier.

Stern, N. (2008), "The economics of climate change", American Economic Review, vol. 98, $\mathrm{N}^{\circ} 2$, Nashville, Tennessee, American Economic Association.

(2007), The Economics of Climate Change, Londres, Cambridge University Press.

Tsigas, M.E., G. Frisvold y B. Kuhn (1997), "Global climate change and agriculture", Global Trade Analysis: Modeling and Applications, T.W. Hertel (ed.), Nueva York, Cambridge University Press.

WRI (Instituto de Recursos Mundiales) (2008), CAIT (Climate Analysis Indicators Tool) database [en línea] http://cait.wri.org.

Zhang, Z.X. (2004), "Meeting the Kyoto targets: The importance of developing country participation", Journal of Policy Modeling, vol. 26, $\mathrm{N}^{\circ} 1$, Amsterdam, Elsevier. 